TRANSACTIONS OF THE

AMERICAN MATHEMATICAL SOCIETY

Volume 358, Number 1, Pages 285-314

S 0002-9947(05)03646-9

Article electronically published on February 18, 2005

\title{
RANDOM FRACTAL STRINGS: THEIR ZETA FUNCTIONS, COMPLEX DIMENSIONS AND SPECTRAL ASYMPTOTICS
}

\author{
B. M. HAMBLY AND MICHEL L. LAPIDUS
}

\begin{abstract}
In this paper a string is a sequence of positive non-increasing real numbers which sums to one. For our purposes a fractal string is a string formed from the lengths of removed sub-intervals created by a recursive decomposition of the unit interval. By using the so-called complex dimensions of the string, the poles of an associated zeta function, it is possible to obtain detailed information about the behaviour of the asymptotic properties of the string. We consider random versions of fractal strings. We show that by using a random recursive self-similar construction, it is possible to obtain similar results to those for deterministic self-similar strings. In the case of strings generated by the excursions of stable subordinators, we show that the complex dimensions can only lie on the real line. The results allow us to discuss the geometric and spectral asymptotics of one-dimensional domains with random fractal boundary.
\end{abstract}

\section{INTRODUCTION}

In 14 there is an extensive analysis of fractal strings. These are sequences extracted from the construction of fractal subsets of $\mathbb{R}$ and are used as models for other infinite series such as the primes. The focus of [14, Chapter 2, was on some simple strings arising from iterated function systems on the real line and, in that setting, by considering an associated zeta function, it is possible to give explicit formulas for various quantities associated with the resulting self-similar string. The explicit formulas are determined by the complex dimensions of the string, defined as the poles of the zeta function.

In this work we will see how some aspects of this theory carry over to the setting of random fractal strings. There are many natural random fractals which generate strings, and we will discuss three. First, a natural generalization of the iterated function system is the random recursive fractal and it generates a corresponding random string. Second, the zero set of Brownian motion provides a fractal set of dimension $1 / 2$ and it too can be used to generate a string. We extend this to strings arising from stable subordinators. We also consider some strings that have larger

Received by the editors October 21, 2003 and, in revised form, February 17, 2004.

2000 Mathematics Subject Classification. Primary 28A80, 60D05; Secondary 11M41, 58J50, $60 \mathrm{~J} 80$.

Key words and phrases. Random recursive fractal, general branching process, fractal string, zeta function, complex dimensions, stable subordinator, tubular neighbourhoods, spectral asymptotics.

The second author was supported in part by the U.S. National Science Foundation under grants DMS-9623002, DMS-0070497. 
random fluctuations, which shows that there is little that can be said in such cases using the techniques developed here.

One reason for the development of the study of fractal strings was that they provide a sufficiently simple set for the study of the second order term in the asymptotics of the eigenvalue counting function of the Laplace operator on a bounded domain. This problem, of hearing the shape of a fractal drum, was considered in a number of papers. See for example [2, 44, 11, [5], 12, [13. In the case of two-dimensional domains with an irregular boundary there is a range of possible behaviour for the second order term in the asymptotics of the eigenvalue counting function. The problem is made simpler if we reduce to one dimension and consider a fractal string as a set with fractal boundary. The explicit formulas that follow from our results on the complex dimensions allow us to obtain precise results on the eigenvalue asymptotics for our random fractal strings.

For example, if we consider the fractal string generated as the complement of the zero set of Brownian motion, then the fractal boundary problem associated to the string has the following property. If we let $N(\lambda)$ denote the eigenvalue counting function (the number of eigenvalues of the Dirichlet Laplace operator on the string whose value is less than $\lambda$ ), then we have the following asymptotic formula; for any $\epsilon>0$, almost surely we have

$$
N(\lambda)=\lambda+L \zeta_{r}(1 / 2) \lambda^{1 / 2}+o\left(\lambda^{1 / 4+\epsilon}\right),
$$

where $L$ is the local time at 0 of Brownian motion run for time 1 and $\zeta_{r}$ is the Riemann zeta function. The error term could be reduced if we could establish meromorphic continuation of the associated zeta function to the left of the region $\{z \in \mathbb{C}: \operatorname{Re}(z)>1 / 4\}$.

An outline of the paper is as follows. We begin by stating some of the results concerning the behaviour of self-similar strings. This is followed by a discussion of the general branching process, a random process which describes the underlying tree structure in a random recursive fractal. We will establish a rate of convergence theorem for such processes; this is required to prove meromorphic continuation of the zeta function of our random recursive strings. In Section 4 we discuss the random recursive strings. In particular, we show that the poles (of the meromorphic continuation) of their zeta functions are almost surely the complex solutions of a natural 'Moran-type' expectation equation. In Section 5 we consider the stable strings and show that their zeta functions can be meromorphically continued and will not have any complex dimensions with non-zero imaginary part. We conclude the discussion of meromorphic continuation by exhibiting in Section 6 a particular type of scale irregular random Cantor set which has large fluctuations, and our techniques do not allow us to prove meromorphic continuation. Finally in Section 7 we discuss some properties of the string that can be deduced from the complex dimensions. These include the asymptotic behaviour of tubular neighbourhoods of the boundary and the asymptotics of the eigenvalue counting function.

\section{SElF-SIMIlar FRACTAL STRINGS AND THEIR ZETA FUNCTIONS}

Fractal strings were introduced in [12, and in [14] a theory of complex dimensions of fractal strings was developed and its connections with number theory explored. We introduce the terminology and several results. Our aim will be to consider 
the zeta functions of our strings to determine their complex dimensions and the behaviour of the zeta function at its poles.

In short, a fractal string is a bounded open subset of the real line, $U \subset \mathbb{R}$, with boundary $F=\partial U$, a totally disconnected (compact) subset of $\mathbb{R}$ with measure 0 . Frequently, a fractal string $U$ is identified with the sequence $\mathcal{L}=\left\{L_{i}\right\}_{i=1}^{\infty}$ of lengths of the connected components (largest open sub-intervals) of $U$, written in nonincreasing order according to multiplicity. To avoid trivial situations it is always assumed that the sequence is infinite and $L_{i} \rightarrow 0$. Without loss of generality it can be assumed that $U \subset[0,1]$ and that $\mathcal{L}$ has total length one: $|U|=\sum_{i=1}^{\infty} L_{i}=1$.

We now focus on self-similar strings; we first recall their construction before discussing some of the results of [14] concerning their complex dimensions. We start with the interval $[0,1]$. A self-similar set can be constructed from a family of similitudes $\left\{\psi_{i}: i=1, \ldots, N\right\}$, where $\psi_{i}:[0,1] \rightarrow[0,1]$, and we write $r_{i}\left(0<r_{i}<\right.$ $1)$ for the Lipschitz constant of $\psi_{i}$. We assume that our maps satisfy the open set condition (OSC) in that there is an open set $O \subset[0,1]$ such that $\psi_{i}(O) \cap \psi_{j}(O)=\emptyset$ and $O \supset \bigcup_{i=1}^{N} \psi_{i}(O)$. The self-similar set $F$ is the unique fixed point in the set of subsets of $[0,1]$ such that

$$
F=\bigcup_{i=1}^{N} \psi_{i}(F) .
$$

It is also obtained as the limit of repeated application of the maps. If we let $I_{1}=$ $\{1, \ldots, N\}$ and set $I_{n}=I_{1}^{n}$ for the sequences of length $n$ we can use this to index the subsets of the fractal. That is, if $\mathbf{i}=\left(i_{1}, \ldots, i_{n}\right)$ we write $\psi_{\mathbf{i}}=\psi_{i_{1}} \circ \psi_{i_{2}} \circ \cdots \circ \psi_{i_{n}}$ and

$$
F=\bigcap_{n=1}^{\infty} \bigcup_{\mathbf{i} \in I_{n}} \psi_{\mathbf{i}}([0,1])
$$

Note that the subsets of the fractal are indexed by an $N$-ary tree.

The set $F$ is a subset of $[0,1]$, and we note that there are subintervals $G_{j} \subset$ $[0,1], j=1, \ldots, M$, such that $G_{j} \cap G_{j^{\prime}}=\emptyset$ for $j \neq j^{\prime} \in\{1, \ldots, M\}$ and

$$
[0,1]=\bigcup_{j=1}^{M} G_{j} \cup \bigcup_{i=1}^{N} \psi_{i}([0,1]) .
$$

The maps which form these intervals are written $\varphi_{i}$, so that $\varphi_{j}([0,1])=G_{j}$. The lengths of the removed intervals $G_{j}$ are denoted by $l_{j}$. It is these removed intervals that we use to form our string. From our recursive construction we see that at each iteration of the maps a new collection of subsets $G_{\mathbf{i} j}=\psi_{\mathbf{i}} \circ \varphi_{j}([0,1])$ is formed with length $\tilde{L}_{\mathbf{i} j}=\prod_{k=1}^{n} r_{i_{k}} l_{j}$. We now place the collection of all lengths in non-increasing order to form our string,

$$
\mathcal{L}=\left(L_{1}, L_{2}, \ldots\right), \quad L_{i} \geq L_{i+1}, \quad \sum_{i=1}^{\infty} L_{i}=1 .
$$

A simple example is the middle third Cantor string in which $M=1, N=2, l=$ $1 / 3, r_{1}=r_{2}=1 / 3$.

The geometric zeta function of the string is given by

$$
\zeta(s)=\sum_{i=1}^{\infty} L_{i}^{s}, \quad s \in \mathbb{C} .
$$


The complex dimensions of the string are defined to be the poles of the meromorphic continuation of the geometric zeta function. A result in [14 is to prove that the geometric zeta functions can be analytically continued to the left of the rightmost pole, which occurs at the dimension of the string and to calculate the residues of the complex dimensions of the string. These give rise to so-called explicit formulas giving more detailed information about this object. In particular, these explicit formulas are expressed in terms of the (visible) complex dimensions and provide a precise description of the oscillatory behaviour in the underlying geometry, dynamics or spectrum.

We will find it convenient to think (as in [14]) of the string as a measure. The sequence $\left(L_{i}\right)_{i=1}^{\infty}$ of ordered lengths defines a measure on $[1, \infty)$ as

$$
\eta(d x)=\sum_{i=1}^{\infty} \delta_{L_{i}^{-1}}(d x)
$$

where $\delta_{x}$ denotes the Dirac measure concentrated at $x$. We will write the counting function as

$$
\eta(x)=\int_{0}^{x} \eta(d y)=\sum_{i=1}^{\infty} I_{\left\{L_{i}^{-1} \leq x\right\}},
$$

where $I_{A}$ denotes the indicator function of the set $A$. Using the expression for the string as a measure we can write the zeta function as a Mellin transform of the measure

$$
\zeta(s)=\int_{0}^{\infty} x^{-s} \eta(d x)
$$

We conclude with a summary of results.

Theorem 2.1. For a self-similar string, the zeta function is given by

$$
\zeta(s)=\frac{\sum_{j=1}^{M} l_{j}^{s}}{1-\sum_{i=1}^{N} r_{i}^{s}} .
$$

The set of complex dimensions is contained in

$$
\mathcal{C}=\left\{s \in \mathbb{C}: \sum_{i=1}^{N} r_{i}^{s}=1\right\} .
$$

The zeta function can be meromorphically continued to the left of the rightmost pole (in fact to all of $\mathbb{C}$ ).

Remark 2.2. (1) The only complex dimension located on the real axis is equal to $d_{f}$, the fractal dimension (Minkowski, upper box dimension) of $F$. It is equal to the similarity dimension of $F$, that is, the unique real solution to the 'Moran equation' $\sum_{i=1}^{N} r_{i}^{s}=1$, and also coincides with the abscissa of convergence of the Dirichlet series defining $\zeta(s)$. Moreover, $d_{f} \in(0,1)$ and is a simple pole of $\zeta(s)$.

(2) In general the complex dimensions are contained in $\mathcal{C}$, as there may be cancellations between the zeros of the numerator and those of the denominator of $\zeta(s)$, as shown in [15]. Of course, in the case of a single gap, $M=1$, then the set of complex dimensions precisely coincides with $\mathcal{C}$. (An analogous statement holds for the lattice case of Theorem 2.3 below.) 
There is a dichotomy in the structure of the complex dimensions. A self-similar string is non-lattice if the numbers $\log r_{i}, i=1, \ldots, N$, are rationally independent, and lattice otherwise. In the lattice case, there exists $r \in(0,1)$, called the 'multiplicative generator' of the string, and positive integers without common divisors, $k_{i}, i=1, \ldots, N$, such that $r_{i}=r^{k_{i}}$. We will write $T=-\log r$. In the lattice case the string has a line of complex dimensions at each zero of the denominator of the zeta function. In the non-lattice case the real parts of the complex dimensions are dense to the left of the rightmost pole of the zeta function. The non-lattice case can be approximated by a sequence of lattice strings whose periods diverge.

Theorem 2.3 (14, Theorem 2.13). In the lattice case $\zeta(s)$ is a rational function of $r^{s}=e^{-T s}$. There are finitely many poles $\omega_{0}:=d_{f}, \omega_{1}, \ldots, \omega_{q}$ of the zeta function (obtained as roots of the polynomial equation $\sum_{i=1}^{N} z^{k_{i}}=1$, with $z=r^{s}$ ) and there is an oscillatory period $p=2 \pi / T$, such that the set of complex dimensions is contained in

$$
\mathcal{C}=\left\{\omega_{u}+\sqrt{-1} n p: n \in \mathbb{Z}, u=0, \ldots, q\right\} .
$$

In the non-lattice case, $d_{f}$ is the unique pole of $\zeta(s)$ on the line $\operatorname{Re}(s)=d_{f}$. The complex dimensions of the string can be approximated by the complex dimensions of a sequence of lattice strings with larger and larger oscillatory period. In particular, there are countably many complex dimensions (a subsequence of which tends to, but does not touch, the line $\operatorname{Re}(s)=d_{f}$ ) and they are all located in a horizontally bounded strip.

One sees that in the lattice case, the complex dimensions are periodically distributed on finitely many lines, whereas in the non-lattice case, they exhibit a 'quasi-periodic behaviour'; the latter is studied in more detail in [14] (see also [15]).

In the final section we will discuss the application of these results to the geometry and spectral properties of the string. By essentially inverting the zeta function we can recover asymptotic properties of various counting functions associated with the string.

\section{The general BRANCHing PRocess}

We will see that random fractal strings display a wider variety of behaviour than their deterministic counterpart. In order to obtain theorems on the complex dimensions analogous to the deterministic case we will need some results from the theory of general or Crump-Mode-Jagers (CMJ) branching processes.

The self-similar set $F$ can be described by the sequence space $I=\bigcup_{n=0}^{\infty} I_{n}$, which we can think of as a tree. In a similar way (to be described below) we think of random recursive fractals as indexed by random trees; see 9 . The sample paths of classical Galton-Watson branching processes therefore provide a description of a random recursive fractal. For a tree which contains more structure we can consider the sample paths of the general branching process or branching random walk.

Let $I_{n}=\bigcup_{k=0}^{n} \mathbb{N}^{k}$ and $I=\bigcup_{k} I_{k}$, where $I_{0}$ is the empty set. The concatenation of sequences $\mathbf{i}$ and $\mathbf{j} \in I$ will be written $\mathbf{i j}$. For an infinite sequence $\mathbf{i} \in I \backslash I_{n}$, denote by $\left.\mathbf{i}\right|_{n}$ the sequence truncated to length $n$ and by $\mathbf{i}[n]$ the $n$-th element of $\mathbf{i}$. We write $\mathbf{j} \leq \mathbf{i}$ if $\mathbf{i}=\mathbf{j k}$ for some $\mathbf{k}$, and denote by $|\mathbf{i}|$ the length of the sequence $\mathbf{i}$.

The general branching process will have a sample space defined by a random subset $T$ of $I$. An element $\mathbf{i} \in \mathbb{N}^{n}$ represents an $n$-th generation individual in the 
branching process. If $\mathbf{i}$ has $j$ children, then these are $\mathbf{i} 1, \mathbf{i} 2, \ldots, \mathbf{i} j$. A tree $T$ is a subset of the space $I$ such that:

i) $\emptyset \in T$ is the root of the tree;

ii) $\mathbf{i} \in T$ implies $\left.\mathbf{i}\right|_{k} \in T$ for all $k<|\mathbf{i}|$;

iii) if $\mathbf{i} j \in T$ for some $j \in \mathbb{N}$, then $\mathbf{i} 1, \mathbf{i} 2, \ldots, \mathbf{i}(j-1) \in T$.

Level $n$ of the tree will be denoted $T_{n}=T \cap \mathbb{N}^{n}$. We have a one-to-one correspondence between trees and feasible realisations of the set of individuals in a branching process.

For the individual indexed by $\mathbf{i}$ we have a description $U_{\mathbf{i}}=\left(\xi_{\mathbf{i}}, \lambda_{\mathbf{i}}, \phi_{\mathbf{i}}\right)$. The $U_{\mathbf{i}}$ are independent and identically distributed and consist of three components. The reproduction process $\xi: \mathbb{R}_{+} \rightarrow \mathbb{Z}_{+}$(where $\mathbb{R}_{+}=[0, \infty), \mathbb{Z}_{+}=0 \cup \mathbb{N}$ ) is a point process describing the offspring produced at each time; $\lambda \in \mathbb{R}_{+}$is the life span, and $\phi$ is a random characteristic, a product-measurable non-negative random process that assigns a score to an individual. Note that we make no assumption about the joint distribution of the components of $U$.

For $t \leq \lambda_{\mathbf{i}}, \xi_{\mathbf{i}}(t)$ is the number of children born to $\mathbf{i}$ in time $t$. We write $N_{\mathbf{i}}=\xi_{\mathbf{i}}\left(\lambda_{\mathbf{i}}\right)$ for the total number of children born to i. Let $T \subset I$ be the individuals in a CMJ process. We take $\emptyset$ as the initial ancestor, and then for each individual $\mathbf{i} \in T$ include its children $\mathbf{i} 1, \mathbf{i} 2, \ldots, \mathbf{i} N_{\mathbf{i}} \in T$. Clearly $T$ is a random tree. Let the birth time of $\emptyset$ be $\sigma_{\emptyset}=0$, and let the birth time for $\mathbf{i} j$ be $\sigma_{\mathbf{i} j}=\sigma_{\mathbf{i}}+t_{\mathbf{i}}(j)$, where $t_{\mathbf{i}}(j)=\inf \left\{t: \xi_{\mathbf{i}}(t) \geq j\right\}$. Any distribution for $U_{\emptyset}$ induces a probability space $(\Omega, \mathcal{B}, \mathbb{P})$, where $\Omega$ is the space of all trees $T$ and their associated $\left\{U_{\mathbf{i}}: \mathbf{i} \in T\right\}$.

Let $Z(t)$ be the population alive at time $t$, given $Z(0)=1$. By considering the offspring of the initial individual $\emptyset$, we have a decomposition of the process as

$$
Z(t)=I_{\left\{\lambda_{\emptyset}>t\right\}}+\sum_{i=1}^{\xi_{\emptyset}(t)} Z_{i}\left(t-\sigma_{i}\right),
$$

where each $Z_{i}$ is an independent copy of the branching process with initial ancestor the individual with address $i$. The process counted with random characteristic $\phi$ is then

$$
Z^{\phi}(t)=\sum_{\mathbf{i} \in T} \phi_{\mathbf{i}}\left(t-\sigma_{\mathbf{i}}\right),
$$

where the $\phi_{\mathbf{i}}$ are i.i.d., and may depend upon the whole process started from i. We note that $Z^{\phi}(t)=Z(t)$ if we use the random characteristic $\phi_{\mathbf{i}}(t)=I_{\left\{0 \leq t<\lambda_{\mathbf{i}}\right\}}$. Let $\mathcal{I}_{s}$ denote the set of sequences corresponding to individuals to be born after time $s$ whose mothers were born before $s$. We can decompose the process with characteristic, at any time $s<t$, as

$$
Z^{\phi}(t)=\sum_{\mathbf{i} \in \mathcal{I}_{s}} \phi_{\mathbf{i}}\left(t-\sigma_{i}\right)+\sum_{\mathbf{i} \in \mathcal{I}_{s}} Z_{\mathbf{i}}^{\phi}\left(t-\sigma_{i}\right) .
$$

We make the following assumptions (writing $\mathbb{E}$ for the expectation under $\mathbb{P}$ ).

Assumption 3.1. (A1) $\mathbb{E} \xi_{\emptyset}(0)=0$, and there exists a unique Malthusian parameter $\alpha \in(0, \infty)$ such that

$$
\mathbb{E} \sum_{i=1}^{N_{\emptyset}} e^{-\alpha \sigma_{i}}=1 \quad \text { and } \quad \mathbb{E} \sum_{i=1}^{N_{\emptyset}} \sigma_{i} e^{-\alpha \sigma_{i}}<\infty .
$$


(A2) For $X_{\alpha}=\sum_{i=1}^{N_{\emptyset}} e^{-\alpha \sigma_{i}}$,

$$
\mathbb{E} X_{\alpha} \log ^{+} X_{\alpha}<\infty .
$$

(A3) There exists on $[0, \infty)$ a non-increasing, positive integrable function $g$ such that $\mathbb{E}\left(\sup _{t} g(t)^{-1} \int_{t}^{\infty} e^{-\alpha u} \xi(d u)\right)<\infty$.

We will assume that our characteristics are bounded.

We note that if $m_{t}^{\phi}=\mathbb{E} e^{-\alpha t} Z_{t}^{\phi}$, then it satisfies a renewal equation

$$
m_{t}^{\phi}=\mathbb{E} e^{-\alpha t} \phi(t)+\int_{0}^{t} m_{t-s} \mu_{\alpha}(d s),
$$

where $\mu_{\alpha}(t)=\mathbb{E} \int_{0}^{t} e^{-\alpha s} \xi(d s)$. This is the distribution function of a probability measure by our choice of $\alpha$. We will refer to a measure as lattice if its support lies on a lattice with period $T$. If the measure is not lattice we call it non-lattice. By the renewal theorem we have that as $t \rightarrow \infty$, if the measure $\mu_{\alpha}$ is non-lattice,

$$
m_{t}^{\phi} \rightarrow m_{\infty}^{\phi}=\frac{\int_{0}^{\infty} \mathbb{E}(\phi(t)) e^{-\alpha t} d t}{\int_{0}^{\infty} t e^{-\alpha t} \mu(d t)} .
$$

In the lattice case we obtain limits down an appropriate subsequence,

$$
m_{n T}^{\phi} \rightarrow \frac{\sum_{j=0}^{\infty} \mathbb{E}(\phi(j T)) e^{-\alpha j T}}{\int_{0}^{\infty} t e^{-\alpha t} \mu(d t)}, \text { as } n \rightarrow \infty .
$$

In either case we will write the limit as $m_{\infty}^{\phi}$.

Let

$$
W_{t}=\sum_{\mathbf{i} \in \mathcal{I}_{t}} e^{-\alpha \sigma_{\mathbf{i}}}
$$

It is shown in 18 , that this process is a martingale and, as it is positive, converges to a limit $W$. We observe that this can be decomposed as

$$
W=\sum_{\mathbf{i} \in \mathcal{I}_{s}} e^{-\alpha \sigma_{\mathbf{i}}} W_{\mathbf{i}}
$$

where $W_{\mathbf{i}}$ denotes the limit random variable started from the individual with address i (and we write $W=W_{\emptyset}$ ).

Under Assumption 3.1 we have the following.

Theorem 3.2 ([18, 7]). Under (A1), (A3), for any characteristic $\phi$ there is a random variable $W$ such that:

(1) If $\mu_{\alpha}$ is non-lattice, then $e^{-\alpha t} Z^{\phi}(t) \rightarrow m_{\infty}^{\phi} W$ almost surely as $t \rightarrow \infty$.

(2) If $\mu_{\alpha}$ is lattice, then $e^{-\alpha n T} Z^{\phi}(n T) \rightarrow m_{\infty}^{\phi} W$ almost surely as $n \rightarrow \infty$.

Moreover, $\mathbb{E} W>0$ if and only if (A2) holds.

Note that in the lattice case we have a finite constant $c_{1}$ such that

$$
\limsup _{t \rightarrow \infty} e^{-\alpha t} Z_{t} \leq c_{1} W .
$$

We will now give two assumptions which each provide a restriction on the branching process.

Assumption 3.3. The probability space $\Omega$ is finite. There are only a finite number of possible life histories $U$.

Assumption 3.4. The reproduction process is bounded in that there exist finite constants $c_{2}$ and $\Lambda_{1}, \Lambda_{2}$ such that $\xi_{\mathbf{i}}\left(\lambda_{\mathbf{i}}\right) \leq c_{2}$ and $\Lambda_{1} \leq \lambda_{\mathbf{i}} \leq \Lambda_{2}$ for all $\mathbf{i} \in T$. 
Clearly Assumption 3.3 will imply Assumption 3.4 and we will therefore make the second assumption unless we require a stronger statement. Both these assumptions imply that the random variable $W$ has moments of all orders (see [3]).

We state here a rate of convergence theorem for the renewal theorem. This will enable us to prove a rate of convergence theorem for the general branching process which will be required to prove the meromorphic continuation of our random recursive strings.

We state the result in a more general form. Let $f, g$ be functions satisfying the renewal equation for a probability measure $\nu$,

$$
f(t)=g(t)+\int_{0}^{t} f(t-s) \nu(d s) .
$$

We will write the first moment as $\bar{\nu}=\int_{0}^{\infty} s \nu(d s)$.

Statements of the rate of convergence in the renewal theorem have been given in a few places. In order to find this theorem we refer the reader to [20; other approaches in the lattice and non-lattice cases can be found in [16, [10]. We call a measure $\nu$ strongly non-lattice if

$$
\liminf _{|\theta| \rightarrow \infty}\left|1-\int_{-\infty}^{\infty} e^{i \theta x} \nu(d x)\right|>0 .
$$

Lemma 3.5. Let $f$ satisfy the renewal equation (3.4) with $g$ a directly Riemann integrable function and $\nu$ a probability measure which has exponential moments, in that $\int_{0}^{\infty} e^{\delta s} \nu(d s)<\infty$ for some $\delta>0$. Then there exists a constant $\rho>0$ such that

(1) In the strongly non-lattice case

$$
\lim _{t \rightarrow \infty} e^{\rho t}\left|f(t)-\frac{1}{\bar{\nu}} \int_{0}^{t} g(s) d s\right|=0 .
$$

(2) In the lattice case, where $\nu$ lies on a lattice with period $T$,

$$
\lim _{t \rightarrow \infty} e^{\rho t}|f(t)-G(t)|=0
$$

where $G$ is the $T$-periodic function given by

$$
G(t)=\frac{T}{\bar{\nu}} \sum_{j=-\infty}^{\infty} g(t+j T) .
$$

Corollary 3.6. Let $g$ be such that $g(t) \leq e^{-\delta t}$ and let $\nu$ be a lattice or strongly non-lattice sub-probability measure in that $\nu$ is a measure such that $\int_{0}^{\infty} \nu(d s)<1$, then there exists a constant $\rho>0$ such that $\int_{0}^{\infty} e^{\rho s} \nu(d s)=1$ and there exists a constant $c_{3}$ such that

$$
f(t) \leq c_{3} e^{-\rho^{\prime} t}
$$

for any $\rho^{\prime}<\min (\delta, \rho)$.

Remark 3.7. (1) We do not have an analogous result for the general non-lattice case.

(2) There are other conditions under which such results can be obtained. In the case of a finite measure (where the strong non-lattice case is the non-lattice case) we refer to [10], where the precise value of $\rho$ is given.

Assumption 3.8. The general branching process mean measure is either lattice or strongly non-lattice. 
Under this assumption, we will let $\rho$ be the rate of convergence in the renewal theorem for the mean of the general branching process,

$$
\rho:=\sup \left\{\beta: \lim _{t \rightarrow \infty} e^{\beta t}\left|m_{t}^{\phi}-m_{\infty}^{\phi}\right|=0\right\} .
$$

This constant could depend upon $\phi$ but as we will only use one particular $\phi$ in what follows, we omit the dependence.

Theorem 3.9. Under Assumptions 3.1, 3.4 and 3.8, for any $0<\kappa<\min (\alpha, \rho) / 2$, then

$$
\lim _{t \rightarrow \infty} e^{-(\alpha-\kappa) t}\left|Z_{t}^{\phi}-W \mathbb{E} Z_{t}^{\phi}\right|=0, \quad \mathbb{P}-a . s .
$$

The proof will follow from a series of lemmas. We begin with the following renewal-type theorem.

Lemma 3.10. Under the assumptions of Theorem 3.9, if a $(t)$ is bounded over finite intervals and satisfies the equation

$$
a(t)=\mathbb{E} \sum_{i=1}^{N_{\emptyset}} e^{-2 \alpha \sigma_{i}} a\left(t-\sigma_{i}\right)+b(t), \quad \forall t>\Lambda_{2},
$$

with $0<|a(0)|<\infty$, where $|b(t)| \leq c_{1} e^{-\gamma t}$ for all $t \geq 0$ for some constant $c_{1}$, then there exists a constant $c_{2}$ such that for all $\gamma^{\prime}<\min (\alpha, \gamma)$,

$$
|a(t)| \leq c_{2} e^{-\gamma^{\prime} t}, \quad \forall t \geq 0
$$

Proof. Fix a $\gamma^{\prime}<\min (\alpha, \gamma)$. As $a(t)$ is finite initially and is bounded over finite intervals we can set $c_{2}=\sup _{t \in\left[0, t_{0}\right]} e^{\gamma^{\prime} t}|a(t)|<\infty$, where $t_{0}$ will be chosen later. Then for $t \in\left[t_{0}, t_{0}+\Lambda_{1}\right]$ we have

$$
\begin{aligned}
|a(t)| & \leq \mathbb{E} \sum_{i=1}^{N_{\emptyset}} e^{-2 \alpha \sigma_{i}} c_{2} e^{-\gamma^{\prime}\left(t-\sigma_{i}\right)}+|b(t)| \\
& \leq c_{2} e^{-\gamma^{\prime} t}\left(\mathbb{E} \sum_{i=1}^{N_{\emptyset}} e^{-\left(2 \alpha-\gamma^{\prime}\right) \sigma_{i}}+c_{1} e^{-\left(\gamma-\gamma^{\prime}\right) t}\right) .
\end{aligned}
$$

Hence, for $\gamma^{\prime}<\alpha$, we know that $C=\mathbb{E} \sum_{i=1}^{N_{\emptyset}} e^{-\left(2 \alpha-\gamma^{\prime}\right) \sigma_{i}}<1$ and hence for $\gamma^{\prime}<\gamma$ we can choose $t_{0}$ such that $C+c_{1} e^{-\left(\gamma-\gamma^{\prime}\right) t_{0}} \leq 1$. Thus

$$
|a(t)| \leq c_{2} e^{-\gamma^{\prime} t}, \quad t \in\left[0, t_{0}+\Lambda_{1}\right] .
$$

Proceeding in the same way we can extend the estimate to $\left[t_{0}+n \Lambda_{1}, t_{0}+(n+1) \Lambda_{1}\right]$ for all integers $n$, giving the result.

Remark 3.11. Even though the equation satisfied by $a(t)$ is a renewal equation, we have chosen to prove this result directly, as it is all we require for the proof of our main theorem.

Now we turn to a variance calculation that will establish Theorem 3.9. Let $\beta_{\mathbf{i}}^{\phi}(t)=e^{-\alpha t} Z_{\mathbf{i}}^{\phi}(t)$, so that $m_{t}^{\phi}=\mathbb{E} \beta_{t}^{\phi}$, and note that $X_{\mathbf{i}}(t)=\beta_{\mathbf{i}}^{\phi}(t)-m_{t}^{\phi} W_{\mathbf{i}}$ has mean 0 . Let $V(t)=\mathbb{E}\left(X^{2}(t)\right)=\mathbb{E}\left(\beta^{\phi}(t)-m_{t}^{\phi} W\right)^{2}$. This quantity is bounded over finite time intervals as, under Assumption 3.4 all the moments of $W$ and $\beta^{\phi}$ exist. 
Lemma 3.12. Under Assumptions 3.1 , 3.4 and 3.8 for any $\gamma<\min (\alpha, \rho)$, there exists a constant $c_{3}$ such that

$$
V(t) \leq c_{3} e^{-2 \gamma t}, \quad t>0 .
$$

Proof. We will consider $s=0+$ in (3.3), so that we are just splitting the population based on the first individual and then $\mathbf{i}=i$ for $i=1, \ldots, N_{\emptyset}$. Thus, for $t>\Lambda_{2}$,

$$
\begin{aligned}
X_{t} & =\sum_{i=1}^{N_{\emptyset}} e^{-\alpha \sigma_{i}} \beta_{t-\sigma_{i}}^{\phi}-\sum_{i=1}^{N_{\emptyset}} e^{-\alpha \sigma_{i}} m_{t}^{\phi} W_{i} \\
& =\sum_{i=1}^{N_{\emptyset}} e^{-\alpha \sigma_{i}} X_{t-\sigma_{i}}+\sum_{i=1}^{N_{\emptyset}} e^{-\alpha \sigma_{i}}\left(m_{t-\sigma_{i}}^{\phi}-m_{t}^{\phi}\right) W_{i}
\end{aligned}
$$

and, by conditioning on the first event, using independence between branches and the fact that $\mathbb{E} X_{t}=0$, we have that

$$
\begin{aligned}
& V(t)= \mathbb{E} X_{t}^{2} \\
&= \mathbb{E}\left(\sum_{i=1}^{N_{\emptyset}} e^{-\alpha \sigma_{i}} X_{t-\sigma_{i}}\right)^{2}+2 \mathbb{E} \sum_{i=1}^{N_{\emptyset}} e^{-\alpha \sigma_{i}} X_{t-\sigma_{i}} \sum_{i=1}^{N_{\emptyset}} e^{-\alpha \sigma_{i}}\left(m_{t-\sigma_{i}}^{\phi}-m_{t}^{\phi}\right) W_{i} \\
& \quad+\mathbb{E}\left(\sum_{i=1}^{N_{\emptyset}} e^{-\alpha \sigma_{i}}\left(m_{t-\sigma_{i}}^{\phi}-m_{t}^{\phi}\right) W_{i}\right)^{2} \\
&=\mathbb{E} \sum_{i=1}^{N_{\emptyset}} e^{-2 \alpha \sigma_{i}} V\left(t-\sigma_{i}\right)+g(t),
\end{aligned}
$$

where

$$
g(t)=\mathbb{E} \sum_{i=1}^{N_{\emptyset}} e^{-2 \alpha \sigma_{i}}\left(m_{t-\sigma_{i}}^{\phi}-m_{t}^{\phi}\right) \mathbb{E}\left(X_{t-\sigma_{i}} W_{i}\right)+\mathbb{E}\left(\sum_{i=1}^{N_{\emptyset}} e^{-\alpha \sigma_{i}}\left(m_{t-\sigma_{i}}^{\phi}-m_{t}^{\phi}\right) W_{i}\right)^{2} .
$$

We need to control $g(t)$ and for this we need to compute $f(t)=\mathbb{E}(W X(t))$ using Lemma 3.10. By splitting at the first individual, for $t>\Lambda_{2}$,

$$
f(t)=\mathbb{E} W \sum_{i=1}^{N_{\emptyset}} e^{-2 \alpha \sigma_{i}} X\left(t-\sigma_{i}\right)+\mathbb{E} W \sum_{i=1}^{N_{\emptyset}}\left(m_{t}^{\phi}-m_{t-\sigma_{i}}^{\phi}\right) e^{-\alpha \sigma_{i}} W_{i} .
$$

This expression can be written, using conditioning, as

$$
\begin{aligned}
f(t) & =\mathbb{E} \sum_{i=1}^{N_{\emptyset}} e^{-\alpha \sigma_{i}} W_{i} \sum_{i=1}^{N_{\emptyset}} e^{-2 \alpha \sigma_{i}}\left(X\left(t-\sigma_{i}\right)-\left(m_{t}^{\phi}-m_{t-\sigma_{i}}^{\phi}\right) W_{i}\right) \\
& =\mathbb{E} \sum_{i=1}^{N_{\emptyset}} e^{-2 \alpha \sigma_{i}} f\left(t-\sigma_{i}\right)+h(t),
\end{aligned}
$$

where

$$
h(t)=\mathbb{E} \sum_{i=1}^{N_{\emptyset}} \sum_{j=1}^{N_{\emptyset}}\left(m_{t-\sigma_{i}}^{\phi}-m_{t}^{\phi}\right) \mathbb{E} W_{i} W_{j} .
$$

By Lemma 3.5 we have constants $C_{1}, C_{2}$ such that

$$
\left|m_{t}^{\phi}-m_{t-\sigma_{i}}^{\phi}\right| \leq\left|m_{t}^{\phi}-m_{\infty}^{\phi}\right|+\left|m_{\infty}^{\phi}-m_{t-\sigma_{i}}^{\phi}\right| \leq C_{1} e^{-\rho t}+C_{2} e^{-\rho\left(t-\sigma_{i}\right)} .
$$


Observing that $\sigma_{i} \leq \Lambda_{2}$ we have

$$
\left|m_{t}^{\phi}-m_{t-\sigma_{i}}^{\phi}\right| \leq C_{3} e^{-\rho t} .
$$

Now, using (3.7), we have

$$
\begin{aligned}
|h(t)| & \leq \mathbb{E} \sum_{i=1}^{N_{\emptyset}} \sum_{j=1}^{N_{\emptyset}}\left|m_{t-\sigma_{i}}^{\phi}-m_{t}^{\phi}\right| \mathbb{E} W_{i} W_{j} \\
& \leq C_{3} e^{-\rho t}\left(\mathbb{E} N_{\emptyset}\left(N_{\emptyset}-1\right)+\mathbb{E} N_{\emptyset} \mathbb{E} W^{2}\right) \\
& \leq C_{4} e^{-\rho t} .
\end{aligned}
$$

As $f$ is bounded over finite intervals, we can now apply Lemma 3.10 to (3.6) to obtain the bound that $|f(t)| \leq C_{5} e^{-\rho^{\prime} t}$ for any $\rho^{\prime}<\min (\alpha, \rho)$. Putting this into the expression for $g(t)$ we have

$$
\begin{aligned}
|g(t)| & \leq \mathbb{E} \sum_{i=1}^{N_{\emptyset}} e^{-2 \alpha \sigma_{i}}\left|m_{t-\sigma_{i}}^{\phi}-m_{t}^{\phi}\right|\left|f\left(t-\sigma_{i}\right)\right|+\mathbb{E}\left(\sum_{i=1}^{N_{\emptyset}} e^{-\alpha \sigma_{i}}\left|m_{t-\sigma_{i}}^{\phi}-m_{t}^{\phi}\right| W_{i}\right)^{2} \\
& \leq C_{3} e^{-\rho t} C_{5} e^{-\rho^{\prime} t}+C_{3} e^{-2 \rho t} \mathbb{E}\left(W^{2}\right) \\
& \leq C_{6} e^{-\left(\rho+\rho^{\prime}\right) t} .
\end{aligned}
$$

Thus, as $V$ is bounded over finite intervals, we can apply Lemma 3.10 once again to obtain the existence of the constant $c_{3}$ such that

$$
V(t) \leq c_{3} e^{-2 \rho^{\prime} t}
$$

for any $\rho^{\prime}<\min (\alpha, \rho)$ as required.

Proof of Theorem 3.9. The almost sure control on $X_{t}$ is then accomplished via Chebychev's inequality and our variance calculation as, for any $\gamma<\min (\alpha, \rho)$,

$$
P\left(X_{t}>\theta\right) \leq \frac{1}{\theta^{2}} V(t) \leq c_{6} \theta^{-2} e^{-\gamma t} .
$$

Hence, for $\epsilon>0$, we can set $\theta=e^{-(\gamma-\epsilon) t / 2}$ to obtain, by Borel-Cantelli, that there is a constant $C$ such that

$$
X_{t} \leq C e^{-(\gamma-\epsilon) t / 2}, \mathbb{P}-a . s .
$$

As this holds for arbitrary $\epsilon$, we have the desired result.

\section{RANDOM RECURSIVE STRINGS}

We now turn to our examples of random fractal strings. The first class is generated by random recursive fractals in one dimension.

Let $A$ be a finite set. For each $a \in A$ we have a set of $N_{a}$ similitudes $\psi^{a}=\left\{\psi_{i}^{a}\right.$ : $\left.i=1, \ldots, N_{a}\right\}$, where $\psi_{i}^{a}:[0,1] \rightarrow[0,1]$ is a map with contraction factor $r_{i}^{a}$ and $\psi^{a}$ satisfies the open set condition (with the same open set) for each $a \in A$. The fractal $F_{a}$ is the unique compact set satisfying

$$
F_{a}=\bigcup_{i=1}^{N_{a}} \psi_{i}^{a}\left(F_{a}\right) .
$$


The string is formed from the sequence of removed subintervals, and for each $a$ we can write

$$
[0,1]=\bigcup_{j=1}^{M_{a}} \varphi_{j}^{a}([0,1]) \cup \bigcup_{i=1}^{N_{a}} \psi_{i}^{a}([0,1]),
$$

where the maps $\varphi^{a}=\left\{\varphi_{j}^{a}: j=1, \ldots, M_{a}\right\}$ are similitudes and $\varphi_{j}^{a}$ has contraction factor $l_{j}^{a}$.

We now wish to combine these into a random recursive fractal string using a probability measure on the set $A$. The informal description is that we start with the unit interval and choose a set of similitudes $\left(\psi^{a}, \varphi^{a}\right)$ according to this probability measure and apply it to the unit interval. We retain the removed lengths as part of the string and then repeat this procedure independently for each copy of the unit interval given by the $\psi_{i}^{a}$. The collection of removed lengths, in non-increasing order, is the random fractal string.

More formally we proceed as follows. In order to construct a random recursive string we need an address space. We use the same address space we used to label the individuals in the general branching process, the set of integer sequences $I$. Let

$$
U_{\mathbf{i}}=\left(N_{\mathbf{i}}, M_{\mathbf{i}}, r_{1}(\mathbf{i}), \ldots, r_{N}(\mathbf{i}), l_{1}(\mathbf{i}), \ldots, l_{M}(\mathbf{i})\right), \mathbf{i} \in I
$$

be independent and identically distributed $\left(\mathbb{N}, \mathbb{N},(0,1)^{\mathbb{N}}\right)$-valued random variables. We define a probability measure $\Phi_{n}$ on $(0,1)^{n}$ and take $\left\{p_{n, m}\right\}_{n, m \in \mathbb{N}}$ to be a probability distribution on $\mathbb{N} \times \mathbb{N}$. Then the probability measure for $U$ is given by

$$
\mathbb{P}\left(U \in\left(n, m, S_{n}\right)\right)=p_{n, m} \Phi_{n+m}\left(S_{n+m}\right), \quad S_{n+m} \subset(0,1)^{n+m}, n \in \mathbb{N} .
$$

A random tree $T$ is a subset of $I$ such that

(i) $\emptyset \in T$ is the root of the tree;

(ii) $\mathbf{i} \in T$ implies $\left.\mathbf{i}\right|_{k} \in T$ for all $k<|\mathbf{i}|$;

(iii) $\mathbf{i} \in T$ implies $\mathbf{i} 1, \mathbf{i} 2, \ldots, \mathbf{i} N(\mathbf{i}) \in T$, and $\mathbf{i}(N(\mathbf{i})+1), \mathbf{i}(N(\mathbf{i})+2), \ldots \notin T$.

We will write $(\Omega, \mathcal{B}, \mathbb{P})$ for the natural probability space associated with these trees. That is, a sample point $\omega \in \Omega$ will denote a random tree $T$ and the associated $\left\{U_{\mathbf{i}}: \mathbf{i} \in T\right\}$. The $\sigma$-algebras are defined as

$$
\mathcal{B}_{n}=\sigma\left(U_{\mathbf{i}} ; \mathbf{i} \in T_{n-1}\right), \quad \mathcal{B}=\bigcup_{n=1}^{\infty} \mathcal{B}_{n}
$$

and the probability measure, $\mathbb{P}$, is as defined previously. If we project this measure onto its first coordinate it is the offspring distribution for a Galton-Watson branching process.

We now regard our random variables $U_{\mathbf{i}}$ with probability measure $\mathbb{P}$ as taking values in the set $A$ of possible families of maps by taking $N_{a}$ as the number of maps in the family $\psi^{a}$ and $M_{a}$ as the number of removed intervals needed for the string. The $\left\{r_{i}^{a}, i=1, \ldots, N\right\}$ and $\left\{l_{j}^{a}, j=1, \ldots, M\right\}$ are the Lipschitz constants of the respective maps. We note that we must have $1 \leq M_{a} \leq N_{a}+1$. From now on we drop the sub or superscript $a$ and regard the $N, M, r_{i}, l_{j}$ as random variables.

The address of each branch in the tree is now used to specify our random Cantor set. Let

$$
C_{\mathbf{i}}=\psi_{\mathbf{i}}([0,1]):=\psi_{\left.\mathbf{i}\right|_{1}}^{U_{\left.\mathbf{i}\right|_{1}}}\left(\cdots\left(\psi_{\left.\mathbf{i}\right|_{n}}^{U_{\left.\mathbf{i}\right|_{n}}}([0,1])\right)\right)
$$


A random recursive fractal can then be defined by

$$
F^{\omega}=\bigcap_{n=1}^{\infty} \bigcup_{\mathbf{i} \in T_{n}(\omega)} C_{\mathbf{i}} .
$$

We now define a specific general branching process $Z$ with the property that at time $t$ the individuals alive in the population each correspond to a set in the fractal of roughly size $e^{-t}$. Let the reproduction process and life span be given by

$$
(\xi, \lambda)=\left(\sum_{i=1}^{N} \delta_{-\log r_{i}}, \quad \max _{1 \leq i \leq N}-\log r_{i}\right),
$$

where $U^{\prime}=\left(N, r_{1}, \ldots, r_{N}\right)$ is chosen according to the appropriate marginal distribution of $\mathbb{P}$. The birth time $\sigma_{\mathbf{i}}$ of an individual with address (ancestry) $\mathbf{i}$ can be written as

$$
\sigma_{\mathbf{i}}=-\log r_{\mathbf{i}}, \text { where } r_{\mathbf{i}}=\prod_{k=1}^{|\mathbf{i}|} r_{\mathbf{i}[k]}\left(\left.\mathbf{i}\right|_{k-1}\right) .
$$

Let $\left(\Omega_{\emptyset}, \mathcal{B}_{\emptyset}, \mathbb{P}\right)$ be the probability space generated by $U_{\emptyset}$. The branching process assumptions of Section 3 can be reexpressed for the fractals as

Assumption 4.1. (A1) $\mathbb{P}\left(r_{1}(\emptyset)=1\right)=0$, and there exists a unique $\alpha \in(0, \infty)$ such that

$$
\mathbb{E} \sum_{i=1}^{N(\emptyset)} r_{i}(\emptyset)^{\alpha}=1 \text { and } \mathbb{E} \sum_{i=1}^{N(\emptyset)}\left|\log r_{i}(\emptyset)\right| r_{i}(\emptyset)^{\alpha}<\infty .
$$

(A2) $\Omega_{\emptyset}$ is finite.

(A3) The $r_{i}$ are bounded away from 0.

(A4) The measure $\mu$, with distribution function

$$
\mu(x)=\mathbb{E} \int_{0}^{x} \sum_{i=1}^{N(\emptyset)} y^{-\alpha} \delta_{-\log r_{i}(\emptyset)} d y,
$$

is either lattice or strongly non-lattice.

Remark 4.2. (1) Under Assumption 4.1(A2), $\Phi_{n}$ is a finite atomic measure on $(0,1)^{n}$.

(2) Under both Assumption 4.1(A2) and (A3) there exist $r_{*}, r^{*}$ such that $0<$ $r_{*} \leq r_{i}(\emptyset, \omega) \leq r^{*}<1$ for all $i=1, \ldots, N(\emptyset, \omega), \omega \in \Omega_{\emptyset}$ and $N(\emptyset, \omega) \leq \bar{N}<\infty$ a.s., for some scalar $\bar{N}$.

(3) Assumption 4.1(A4) gives the lattice/non-lattice dichotomy for our strings, that is, it is lattice if $\log r_{i}^{a}$ are rationally related for all $i=1, \ldots, N_{a}, a \in A$. In particular we see that if the probability measure has a density on $A$, we cannot have a lattice case.

The Hausdorff dimension of the set $F^{\omega}$ can be found by applying the results of [6], 17] and, as we have assumed the OSC, it is given by the Malthusian parameter of the CMJ process,

$$
d_{f}\left(F^{\omega}\right)=\inf \left\{\alpha \geq 0: \mathbb{E}\left(\sum_{i=1}^{N(\emptyset)} r_{i}^{\alpha}(\emptyset)\right)=1\right\}, \text { for } \mathbb{P} \text {-a.e. } \omega \in \Omega .
$$

Note that for these fractals the other natural notions of dimension, Minkowski, upper box, etc. all coincide with the Hausdorff dimension. 
Now we describe the construction of the random fractal string. To begin we construct the sample path of this process as our random tree. Associate with each vertex of the tree the set of removed lengths $\left(l_{j}: j=1, \ldots, M\right)$ of the type of offspring generated from that vertex. The lengths that compose the random fractal string are then given by $L_{\mathbf{i} j}^{\prime}=\prod_{j=1}^{n-1} r_{i_{j}} l_{j}$ at vertex $\mathbf{i}=\left(i_{1}, \ldots, i_{n}\right)$. We then write them in length order to obtain the string $\mathcal{L}=\left(L_{i}\right)_{i=1}^{\infty}$.

As in Section 2 we think of the string as a measure, and for the random string it will be a random measure. Our general branching process can be used to encode this random measure $\eta$ by use of a suitable characteristic. We set

$$
\phi_{i}(t)=\sum_{j=1}^{M_{i}} I_{\left\{-\log l_{j} \leq t\right\}} .
$$

That is, the lengths of the removed pieces of string are a function of the individual representing that application of the similitude. We also only count the removed part if it is sufficiently large. We can check that with this choice our general branching process has encoded the random measure $\eta$.

By the definition of the general branching process and the counting function $\eta$ in (2.1), we have

$$
\begin{aligned}
z_{t}^{\phi} & =\sum_{\mathbf{i} \in \mathcal{I}_{\infty}} \phi\left(t-\sigma_{\mathbf{i}}\right) \\
& =\sum_{\mathbf{i} \in \mathcal{I}_{\infty}} \sum_{j=1}^{M_{\mathbf{i}}} I_{\left\{-\log l_{j} \leq t-\sigma_{\mathbf{i}}\right\}} \\
& =\sum_{\mathbf{i} \in \mathcal{I}_{\infty}} \sum_{j=1}^{M_{\mathbf{i}}} I_{\left\{L_{\mathbf{i} j}^{-1} \leq e^{t}\right\}} \\
& =\sum_{i=1}^{\infty} I_{\left\{L_{i}^{-1} \leq e^{t}\right\}}=\eta\left(e^{t}\right) .
\end{aligned}
$$

We note that, as all lengths are less than one, the branching process begins at $t=0$ when $Z(0)=1$ and $z_{0}^{\phi}=0$.

We can then use our Theorem $[3.2$, concerning random characteristics, to determine the growth rate of the string.

Corollary 4.3. Under Assumption 4.1 there is a mean one random variable $W$ such that in the non-lattice case

$$
\lim _{t \rightarrow \infty} e^{-\alpha t} \eta\left(e^{t}\right)=m_{\alpha}^{\eta}(\infty) W, \mathbb{P} \text {-a.s. }
$$

and in the lattice case

$$
\lim _{n \rightarrow \infty} e^{-\alpha n T} \eta\left(e^{n T}\right)=m_{\alpha}^{\eta}(\infty) W, \quad \mathbb{P}-a . s .
$$

By using the fact that the quantity $m_{\alpha}^{\eta}(\infty)$ is the limit of the normalized mean growth rate we can write, in the non-lattice case,

$$
\lim _{t \rightarrow \infty} \frac{\eta\left(e^{t}\right)}{\mathbb{E} \eta\left(e^{t}\right)}=W, \quad \mathbb{P}-a . s .
$$

We have a similar expression in the lattice case down a subsequence. The random measure $\eta$ will satisfy a distributional equality in that it can be expressed as a sum 
of scaled copies of itself,

$$
\eta(d x)=\sum_{j=1}^{M} \delta_{l_{j}^{-1}}+\sum_{i=1}^{N} \eta\left(r_{i} d x\right) .
$$

The zeta function of the random string is a random function defined as the Mellin transform of the measure $\eta$,

$$
\zeta(s)=\int_{0}^{\infty} x^{-s} \eta(d x) .
$$

The complex dimensions of the random fractal string are the poles of this function. In order to compute them we consider the mean zeta function which is given by

$$
\mathbb{E} \zeta(s)=\int_{0}^{\infty} x^{-s} \mathbb{E} \eta(d x) .
$$

Lemma 4.4. The mean geometric zeta function can be expressed as

$$
\mathbb{E} \zeta(s)=\frac{\mathbb{E} \sum_{j=1}^{M} l_{j}^{s}}{1-\mathbb{E}\left(\sum_{i=1}^{N} r_{i}^{s}\right)} .
$$

If Assumption 4.1(A2) holds, the family size is finite, then the mean zeta function can be meromorphically continued to $\mathbb{C}$. If Assumption 4.1(A3) holds, then the mean zeta function can be meromorphically continued up to the line $\operatorname{Re}(s)=0$. In either case, $\mathbb{E} \zeta(s)$ is given by the above expression for $s$ in the indicated region.

Proof. We can use the branching structure of the random set to find a closed form expression for this mean zeta function. Observe that by construction we have the following equality in distribution:

$$
\zeta(s)=\sum_{j=1}^{M} l_{j}^{s}+\sum_{i=1}^{N} r_{i}^{s} \zeta_{i}(s),
$$

where the $\zeta_{i}$ are i.i.d. copies of the original zeta function and the random variables $l_{j}, r_{i}$ are determined by the particular choice of $a \in A$ corresponding to the first individual.

If we take expectations in (4.2) and condition on the first map,

$$
\mathbb{E} \zeta(s)=\mathbb{E} \sum_{j=1}^{M} l_{j}^{s}+\mathbb{E}\left(\sum_{i=1}^{N} r_{i}^{s} \mathbb{E} \zeta_{i}(s)\right) .
$$

As the $\zeta_{i}$ are just copies of $\zeta$ we can rearrange to get

$$
\mathbb{E} \zeta(s)=\frac{\mathbb{E} \sum_{j=1}^{M} l_{j}^{s}}{1-\mathbb{E}\left(\sum_{i=1}^{N} r_{i}^{s}\right)} .
$$

The poles of this function lie in the set of $s \in \mathbb{C}$ such that $\mathbb{E}\left(\sum_{i=1}^{N} r_{i}^{s}\right)=1$.

Under Assumption 4.1(A2) the probability space is finite and we have the following explicit formula for the mean zeta function as written in (4.3):

$$
\mathbb{E} \zeta(s)=\frac{\sum_{a \in A} \sum_{j=1}^{M_{a}} l_{j}^{a}}{1-\sum_{a \in A} \sum_{i=1}^{N_{a}}\left(r_{i}^{a}\right)^{s} p_{a}} .
$$

As the sums are finite $\mathbb{E} \zeta(s)$ can be meromorphically continued to the whole of the complex plane. 
Under Assumption 4.1(A3) the expectations in (4.3) can be computed for $R e(s)$ $>0$ and hence $\mathbb{E} \zeta(s)$ can be continued to this region.

The expression for the poles is exactly the expression for the dimension of the boundary of the fractal string and hence we see that the zeta function is well defined for $\operatorname{Re}(s)>d_{f}$. We now wish to show that the random zeta function can be meromorphically continued to the left of this line.

The approach we take is based on the Euler-Maclaurin series approach to proving meromorphic continuation for the usual Riemann zeta function. From the discussion prior to Lemma 4.4 we can write

$$
\zeta(s)=W \mathbb{E} \zeta(s)+\int_{0}^{\infty} x^{-s} V(d x), \quad \mathbb{P}-a . s .,
$$

where $W$ is the limiting random variable for the normed limit of the distribution function for the measure $\eta$ and $V(d x)=(\eta-W \mathbb{E}(\eta))(d x)$ is a random signed measure. In order to extend our zeta function we need to be able to compute the second term for suitable $s \in \mathbb{C}$ and for this purpose we look at the behaviour of

$$
V(x)=\int_{0}^{x} V(d y)=\eta(x)-W \mathbb{E}(\eta(x)) .
$$

The fluctuations in this function follow from those of the corresponding general branching process derived in Section 3.

We can now use this result to establish our main theorem.

Theorem 4.5. Under Assumptions 4.1(A1), (A3), (A4), there is a number $\tau$ with $0<\tau<\min (\alpha, \rho) / 2$ such that the random zeta function $\zeta(s)$ can be meromorphically extended to the right half-plane $\mathcal{D}:=\{s: \operatorname{Re}(s)>\alpha-\tau\}$, where $\alpha$ is given in Assumption 4.1(A1) above. The poles of the random zeta function lie in the set of complex solutions $z \in \mathcal{D}$ to the equation

$$
\mathbb{E}\left(\sum_{i=1}^{N} r_{i}^{z}\right)=1
$$

The residues at the poles $z$ (when the latter are simple) are given by

$$
W \operatorname{Res}(\mathbb{E}(\zeta(s)) ; s=z),
$$

where $\mathbb{E} \zeta(s)$ is the mean zeta function given in Lemma 4.4 .

Proof. With our Theorem 3.9 we immediately deduce the meromorphic extension from (4.4). This follows if we can prove the existence of the integral $\int_{0}^{\infty} x^{-s} V(d x)$. By construction it is a question of determining the behaviour of $V$ at $\infty$ and, by Theorem 3.9, there is a random constant $C$ and a $0<\tau<\min (\alpha, \rho) / 2$ (where $\rho$ is the rate of convergence in the renewal theorem for the branching process mean) such that $V(y) \leq C y^{\alpha-\tau}$ for large $y$. Hence $\int_{0}^{\infty} x^{-s} V(d x)$ is well defined for $\operatorname{Re}(s)>\alpha-\tau$.

We immediately see that

$$
\zeta(s)=W \frac{\mathbb{E} \sum_{j=1}^{M} l_{j}^{s}}{1-\mathbb{E}\left(\sum_{i=1}^{N} r_{i}^{s}\right)}+\int_{0}^{\infty} x^{-s} V(d x),
$$


so that the poles of $\zeta(s)$ in $\mathcal{D}$ occur at the same place as those of $\mathbb{E}(\zeta)$. To compute the residues we again use the formula (4.4).

$$
\begin{aligned}
\operatorname{Res}(\zeta(s) ; s=z) & =\lim _{s \rightarrow z}(s-z) \zeta(s) \\
& =W \operatorname{Res}(\mathbb{E}(\zeta(s)) ; s=z)+\lim _{s \rightarrow z}(s-z) \int_{0}^{\infty} x^{-s} V(d x) \\
& =W \operatorname{Res}(\mathbb{E}(\zeta(s)) ; s=z),
\end{aligned}
$$

as desired.

Remark 4.6. (1) Note that in general there may be cancellations between the zeros of the numerator $\mathbb{E}\left(\sum_{j=1}^{M} l_{j}^{s}\right)$ and those of the denominator $1-\mathbb{E}\left(\sum_{i=1}^{N} r_{i}^{s}\right)$ of the mean zeta function. In the deterministic case examples of such cancellations can be found in [15. However if $M=1$, the poles of the random zeta function $\zeta(s)$ are precisely located at the complex solutions of $\mathbb{E}\left(\sum_{i=1}^{N} r_{i}^{z}\right)=1$ (such that $\operatorname{Re}(z)>$ $\alpha-\tau)$.

(2) In the deterministic case, the poles may have multiplicity greater than one 14], Section 2.2.3; the corresponding Laurent series of the zeta function can, however, be used instead. In the random case, the above proof shows that the Laurent series of $\zeta(s)$ at a pole $z$ can be expressed in terms of that of $\mathbb{E} \zeta(s)$ at $z$.

\section{StABle STRINGS}

In this section we will discuss the case where the string is generated by the excursions of a stable subordinator. The main example to bear in mind is the Brownian string. One-dimensional Brownian motion run for a unit time can be decomposed into a countable family of excursions away from the origin. The local time of Brownian motion at 0 can be defined as the density of the occupation measure of the process at 0 . We can then regard the Brownian path as a Poisson process of excursions indexed by the local time process. Our string will be constructed as the series of ranked excursion lengths, and as the time at the origin has 0 Lebesgue measure, it will have the property of total unit length. Our aim will be to consider stable strings, establish the asymptotic behaviour of the string, show that its zeta function can be meromorphically continued to the left of its rightmost pole and obtain an expression for the zeta function at the poles. Our main conclusion is that for such strings the complex dimensions are purely real.

5.1. Stable subordinators. The ranked lengths of the excursions of stable subordinators are known to have a Poisson-Dirichlet, $\operatorname{PD}(\alpha, 0)$ distribution for $0<\alpha<1$. This type of distribution initially arose when defining the relative frequencies of various species in a large population. There are a number of ways of defining such distributions and we refer to [19] for an extensive survey. For our purposes we recall the definition through subordination and the construction through a family of independent identically distributed random variables.

Let $\tau_{s}$ be a stable subordinator. This is an increasing process with stationary independent increments which can be characterised via its Laplace transform

$$
\mathbb{E}\left[\exp \left(-\lambda \tau_{s}\right)\right]=\exp \left(-s C \Gamma(1-\alpha) \lambda^{\alpha}\right),
$$

where $C$ is a constant and $\Gamma$ is the Gamma function. Let $V_{1}(T) \geq V_{2}(T) \geq \ldots$ denote the ranked lengths of components of the set $[0, T] \backslash Z$, where $Z$ is the closure 
of the range of $\left\{\tau_{s} ; s \geq 0\right\}$. A basic proposition is that for every $s>0$,

$$
\left(\frac{V_{1}\left(\tau_{s}\right)}{\tau_{s}}, \frac{V_{2}\left(\tau_{s}\right)}{\tau_{s}}, \ldots\right)
$$

has the $\operatorname{PD}(\alpha, 0)$ distribution, and also for fixed times $t>0$,

$$
\left(\frac{V_{1}(t)}{t}, \frac{V_{2}(t)}{t}, \ldots\right)
$$

has the $\mathrm{PD}(\alpha, 0)$ distribution.

From now on we consider the case $t=1$ and write $\left(V_{1}, V_{2}, \ldots\right)$, where $\sum_{i=1}^{\infty} V_{i}=$ 1 , for a random sequence with the $\operatorname{PD}(\alpha, 0)$ distribution. An observation which can be found in [19] is that the random variables $R_{n}=V_{n+1} / V_{n}$ have beta distribution $\beta(n \alpha, 1)$ and are mutually independent. For completeness we recall that the $\beta(u, v)$ distribution has density function

$$
f_{u, v}(x)=\frac{\Gamma(u+v)}{\Gamma(u) \Gamma(v)} x^{u-1}(1-x)^{v-1}, \quad x \in[0,1] .
$$

Thus, given $V_{1}$ we can recursively construct the relative values of the lengths in the string and then determine $V_{1}$ by normalization.

We also note here a first limit result and an important representation.

Lemma 5.1 ([19, Proposition 10). If $\left(V_{n}\right)$ has the $P D(\alpha, 0)$ distribution, then

(1) The limit $L=\lim _{n \rightarrow \infty} n V_{n}^{\alpha}$ exists almost surely.

(2) Let $X_{n}=L V_{n}^{-\alpha}$. Then the points $\left(X_{n}\right)$ are the points of a Poisson random measure with Lebesgue measure as its intensity.

We note that the second part of the lemma means that we can write $X_{n}=$ $\sum_{i=1}^{n} \xi_{i}$, where the $\xi_{i}$ are independent random variables with the exponential distribution with mean 1 .

Once we have defined the ranked excursions we wish to consider the associated zeta function

$$
\zeta_{\alpha}(s)=\sum_{n=1}^{\infty} V_{n}^{s}
$$

The key tool will again be the random measure $\eta$ on $[1, \infty)$ determined from the string by

$$
\eta(d x)=\sum_{n=1}^{\infty} \delta_{V_{n}^{-1}}(d x)
$$

5.2. The mean zeta function. The first step in our analysis is to consider the mean behaviour of the zeta function. We write $\mathbb{P}_{\alpha, \theta}$ for the general $\operatorname{PD}(\alpha, \theta)$ distribution and $\mathbb{E}_{\alpha, \theta}$ for the expectation with respect to this distribution. When $\theta=0$ we will write $\mathbb{P}_{\alpha}$ and $\mathbb{E}_{\alpha}$ for the distribution and its expectation, respectively.

We begin with a useful formula from [19] derived from size biased sampling from the distribution.

Lemma 5.2 ([19], Corollary 3). For the general $P D(\alpha, \theta)$ distribution we have

$$
\mathbb{E}_{\alpha, \theta} \sum_{n=1}^{\infty} f\left(V_{n}\right)=\frac{\Gamma(\theta+1)}{\Gamma(\theta+\alpha) \Gamma(1-\alpha)} \int_{0}^{1}(1-u)^{\alpha+\theta-1} u^{-\alpha-1} f(u) d u .
$$


From this we can easily compute the expected value of the zeta function directly,

$$
\mathbb{E}_{\alpha} \zeta_{\alpha}(s)=\mathbb{E}_{\alpha, 0} \sum_{n=1}^{\infty} V_{n}^{s} .
$$

Setting $f(x)=x^{s}$ in (5.1) and integrating we obtain

$$
\mathbb{E}_{\alpha} \zeta_{\alpha}(s)=\frac{\Gamma(s-\alpha)}{\Gamma(1-\alpha) \Gamma(s)} .
$$

The Gamma function can be analytically continued to the whole complex plane. It has no zeros and has simple poles that lie on the real axis at $0,-1,-2, \ldots$ As the mean zeta function is a ratio of Gamma functions, we know that the poles lie on the real line at the values $\alpha, \alpha-1, \alpha-2, \ldots$ and we have the following lemma.

Lemma 5.3. The mean zeta function can be meromorphically continued to the whole complex plane and is given there by (5.2).

We will also be interested in the mean growth of our measure $\eta$. This can be computed from (5.1) as

$$
\begin{aligned}
\mathbb{E}_{\alpha} \eta(x) & =\mathbb{E}_{\alpha} \int_{1}^{x} \eta(d y) \\
& =\mathbb{E}_{\alpha} \sum_{n=1}^{\infty} I_{\left\{V_{n} \geq 1 / x\right\}} \\
& =\frac{(x-1)^{\alpha}}{\Gamma(\alpha+1) \Gamma(1-\alpha)} \\
& =\frac{\sin \pi \alpha}{\pi \alpha}(x-1)^{\alpha},
\end{aligned}
$$

where the last result follows from the fundamental identities for the Gamma function.

5.3. The zeta function for the string. Finally we consider the actual zeta function associated with the string. The first stage is to prove that it can be meromorphically continued to the left of the line $\operatorname{Re}(s)=\alpha$ and to do this we will use the following.

Theorem 5.4. For all $0<\alpha<1$ we have

$$
\lim _{x \rightarrow \infty} \frac{\eta(x)}{\mathbb{E}_{\alpha}(\eta(x))}=\frac{\pi \alpha}{\sin \pi \alpha} L, \mathbb{P}_{\alpha} \text {-a.s. }
$$

Proof. The key to the result, and to some of the later ones, is that we can obtain limit information about $\eta$ from the asymptotics of $V_{n}$.

We can write

$$
\begin{aligned}
\eta(x) & =\sum_{n=1}^{\infty} I_{\left\{V_{n}^{-1} \leq x\right\}} \\
& = \begin{cases}0 & x<V_{1}^{-1} \\
m & V_{m}^{-1} \leq x<V_{m+1}^{-1}\end{cases}
\end{aligned}
$$

Thus

$$
x^{-\alpha} \eta(x)=\sum_{n=1}^{\infty} x^{-\alpha} n I_{\left\{V_{n}^{-1} \leq x<V_{n+1}^{-1}\right\}}
$$


and

$$
\sum_{n=1}^{\infty} n V_{n+1}^{\alpha} I_{\left\{V_{n}^{-1} \leq x<V_{n+1}^{-1}\right\}}<x^{-\alpha} \eta(x) \leq \sum_{n=1}^{\infty} n V_{n}^{\alpha} I_{\left\{V_{n}^{-1} \leq x<V_{n+1}^{-1}\right\}} .
$$

Now, as $V_{n}^{-1} \rightarrow \infty$ as $n \rightarrow \infty$, we see that taking limits as $x \rightarrow \infty$ is equivalent to taking limits as $n \rightarrow \infty$ and gives, by Lemma 5.1 .

$$
\lim _{x \rightarrow \infty} \sum_{n=1}^{\infty} n V_{n}^{\alpha} I_{\left\{V_{n}^{-1} \leq x<V_{n+1}^{-1}\right\}}=\lim _{n \rightarrow \infty} n V_{n}^{\alpha}=L, \mathbb{P}_{\alpha} \text {-a.s. }
$$

For the left-hand side the change to $V_{n+1}$ does not alter the limit and hence, as upper and lower bounds have the same limit, we have that $\mathbb{P}_{\alpha}$-almost surely $\lim _{x \rightarrow \infty} x^{-\alpha} \eta(x)=L$. We now obtain the stated result by substituting in the function $\mathbb{E}_{\alpha} \eta(x)$ from (5.3).

In order to establish the meromorphic continuation we require a rate of convergence in the above limit theorem. In order to do this we will use the second part of Lemma 5.1. As we can write $L V_{n}^{-\alpha}$ as a sum of independent and identically distributed random variables, we can use the independence to control the growth rate of $L V_{n}^{-\alpha}-n$. We need two preliminary results.

Lemma 5.5. For $\epsilon>0$

$$
\lim _{n \rightarrow \infty} n^{-(1+\epsilon) / 2}\left|L V_{n}^{-\alpha}-n\right|=0, \quad \mathbb{P}_{\alpha} \text {-a.s. }
$$

Proof. We begin by observing that

$$
L V_{n}^{-\alpha}-n=\sum_{i=1}^{n}\left(\xi_{i}-1\right)
$$

where the $\xi_{i}$ are independent exponential random variables with mean 1 . We use a standard large deviation approach

$$
\begin{aligned}
\mathbb{P}_{\alpha}\left(\sum_{i=1}^{n}\left(\xi_{i}-1\right)>x\right) & \leq \mathbb{P}_{\alpha}\left(\exp \left(\theta \sum_{i=1}^{n} \xi_{i}\right)>e^{\theta(x+n)}\right) \\
& \leq e^{-\theta(x+n)} \mathbb{E}_{\alpha}\left(e^{\theta X}\right)^{n} \\
& =e^{-\theta(x+n)}(1-\theta)^{-n}
\end{aligned}
$$

As this holds for all $\theta<1$ we can optimize the bound by setting $\theta=x /(x+n)$ to get

$$
\mathbb{P}_{\alpha}\left(\sum_{i=1}^{n}\left(\xi_{i}-1\right)>x\right) \leq e^{-x}\left(1+\frac{x}{n}\right)^{n} \leq e^{-\frac{1}{2} \frac{x^{2}}{n}\left(1-\frac{2}{3} \frac{x}{n}\right)} .
$$

We can follow the same line of argument to show that

$$
\mathbb{P}_{\alpha}\left(\sum_{i=1}^{n}\left(1-\xi_{i}\right)>x\right) \leq e^{-\frac{1}{2} \frac{x^{2}}{n}} .
$$

Hence, if $x<\frac{1}{2} n$, then in (5.5) we have $1-\frac{2}{3} \frac{x}{n} \geq \frac{2}{3}$ and combining this with (5.6), we arrive at

$$
\mathbb{P}_{\alpha}\left(\left|\sum_{i=1}^{n}\left(1-\xi_{i}\right)\right|>x\right) \leq 2 e^{-\frac{1}{3} \frac{x^{2}}{n}}
$$


Thus for $x=\delta n^{(1+\epsilon) / 2}$, we have summability of the probabilities and hence the first Borel-Cantelli lemma shows that

$$
\limsup _{n \rightarrow \infty} \frac{\left|L V_{n}^{-\alpha}-n\right|}{n^{\frac{1}{2}(1+\epsilon)}} \leq \delta, \quad \mathbb{P}_{\alpha} \text {-a.s. }
$$

As this holds for every $\delta>0$, the limit exists as required.

We are now ready to state our convergence rate result. For convenience we write $\tilde{L}=(\pi \alpha / \sin \pi \alpha) L$.

Theorem 5.6. For any $\epsilon>0$,

$$
\lim _{x \rightarrow \infty} x^{\alpha(1-\epsilon) / 2}\left|\frac{\eta(x)}{\mathbb{E}_{\alpha} \eta(x)}-\tilde{L}\right|=0, \quad \mathbb{P}_{\alpha} \text {-a.s. }
$$

Proof. The proof is an application of Lemma 5.5. It is enough to show that, for any $\delta>0$,

$$
\limsup _{x \rightarrow \infty} x^{\alpha(1-\epsilon) / 2}\left|x^{-\alpha} \eta(x)-L\right| \leq \delta .
$$

As in the proof of the almost sure convergence in Theorem 5.4 we can write

$$
\begin{aligned}
x^{\alpha(1-\epsilon) / 2}\left|x^{-\alpha} \eta(x)-L\right| & =x^{-\alpha(1+\epsilon) / 2}\left|\eta(x)-L x^{\alpha}\right| \\
& =x^{-\alpha(1+\epsilon) / 2}\left|n-L x^{\alpha}\right| I_{\left\{V_{n}^{-1} \leq x<V_{n+1}^{-1}\right\}} \\
& \leq V_{n+1}^{\alpha(1+\epsilon) / 2} \max \left(\left|n-L V_{n}^{-\alpha}\right|,\left|n-L V_{n+1}^{\alpha}\right|\right) I_{\left\{V_{n}^{-1} \leq x<V_{n+1}^{-1}\right\}} .
\end{aligned}
$$

To use Lemma 5.5 we note that

$$
\limsup _{n \rightarrow \infty} \frac{\left|L V_{n}^{-\alpha}-n\right|}{V_{n+1}^{-\alpha(1+\epsilon) / 2}}=\limsup _{n \rightarrow \infty} \frac{\left|L V_{n}^{-\alpha}-n\right|}{n^{(1+\epsilon) / 2}} \lim _{n \rightarrow \infty}\left(n V_{n+1}^{\alpha}\right)^{(1+\epsilon) / 2} .
$$

Thus, applying the result we established in Lemma 5.5. and using the almost sure convergence of $n V_{n}^{\alpha} \rightarrow L$ as $n \rightarrow \infty$, we have the result.

Therefore we have shown that for any $\epsilon>0, \mathbb{P}_{\alpha}$-almost surely, for a constant $C>0$ we have

$$
V(x)=\eta(x)-\tilde{L} \mathbb{E}_{\alpha}(\eta(x)) \leq C x^{\alpha(1+\epsilon) / 2} .
$$

Hence we can use the Euler-Maclaurin approach, as outlined in the proof of Theorem 4.5 in the random recursive fractal case, to show that the zeta function can be meromorphically continued beyond the line $\operatorname{Re}(s)=\alpha$ to the set $\{s: \operatorname{Re}(s)>\alpha / 2\}$. From the properties of the Gamma function we see that the complex dimensions for these strings are purely real and we can state our final theorem for this section. (Note that the Brownian string corresponds to the case $\alpha=1 / 2$.)

Theorem 5.7. The geometric zeta function for the stable string is defined meromorphically for all $s: \operatorname{Re}(s)>\alpha / 2$ and has a simple pole at the value $s=\alpha$ and no other visible poles. The value of the residue at the pole is $\alpha L$.

Remark 5.8. The stable bridge is a stable process with the property that it is conditioned to return to 0 at time 1. It is known [19] that such a process has law $\operatorname{PD}(\alpha, \alpha)$ and hence its mean zeta function can be calculated using (5.10) and has a meromorphic continuation to the whole complex plane. It also has a random recursive structure as indicated in 7], as we can decompose the Cantor set formed by the visits to zero at the first and last zeros either side of $1 / 2$. Thus we can also regard this string as a random recursive string but from an uncountable family 
which does not have scaling ratios bounded away from 0 (as the law of the first return time before $1 / 2$ has support on the interval $[0,1 / 2]$ ). As yet we have no theorem that enables us to prove meromorphic continuation. If this can be done, we can obtain the same results as for the stable string. The poles of the zeta function occur at $\alpha, \alpha-1, \ldots$ and hence there are no non-real complex dimensions. The only change is that the residue at $s=\alpha$ is different. This demonstrates that the class of random recursive strings includes strings that are quite different from deterministic self-similar strings.

\section{A HOMOGENEOUS RANDOM STRING}

We discuss a spatially homogeneous string arising from a random Cantor set. This shows that there are random strings for which the techniques we have employed here to prove meromorphic continuation break down. This example is a simplification of the construction of the random recursive fractals. In this case we do not choose a new family of similitudes for each interval, instead we choose the same family for all sets of a given size. Random fractals of this type have been discussed in 1]. This has the effect of introducing much larger fluctuations in the string and for this reason we are not able to control the extra term in the Euler-Maclaurin approach.

We begin by defining a scale irregular random Cantor set. We let $\psi^{a}=\left\{\psi_{1}^{a}, \psi_{2}^{a}\right\}$ denote the family of two similitudes given for any $0<a<1 / 2$ by

$$
\psi_{1}(x)=a x, \quad \psi_{2}(x)=1-a(1-x) .
$$

Thus the effect of applying the map $\psi^{a}$ to the unit interval is to produce two intervals of length $a$ with a gap of length $1-2 a$ between them. For a given sequence $\mathbf{a}=\left\{a_{i}\right\}_{i=1}^{\infty}$, where $0<a_{i}<1 / 2$ for all $i$, we can define a scale irregular Cantor set as

$$
C_{\mathbf{a}}=\bigcap_{n=1}^{\infty} \bigcup_{\mathbf{i} \in\{1,2\}^{n}} \psi_{i_{1}}^{a_{1}} \circ \cdots \circ \psi_{i_{n}}^{a_{n}}([0,1]) .
$$

Note that if we write $\sigma(\mathbf{a})=\left(a_{2}, a_{3} \ldots\right)$ for the shift map on one-sided sequences, then

$$
C_{\mathbf{a}}=\psi^{a_{1}}\left(C_{\sigma(\mathbf{a})}\right) .
$$

At this stage we make no assumption about the sequence. We can define homogeneous random Cantor sets by generating the sequence a using a probability measure.

Theorem 6.1. The Hausdorff dimension and lower box counting dimension of the scale irregular Cantor set $C_{\mathbf{a}}$ are given by

$$
d_{l b}=\operatorname{dim}_{H}=\liminf _{n \rightarrow \infty} \frac{\log \prod_{i=1}^{n} a_{i}}{n \log 2} .
$$

The packing dimension and upper box counting (Minkowski) dimensions are

$$
d_{u b}=\operatorname{dim}_{P}=\limsup _{n \rightarrow \infty} \frac{\log \prod_{i=1}^{n} a_{i}}{n \log 2} .
$$

Proof. This is the same as the case of scale irregular Sierpinski gaskets as given in [1]. 
Our next step is to describe the scale irregular string that is determined by this scale irregular Cantor set. From this point we assume for convenience that $a_{n}<1 / 3$ for all $n$. This assumption ensures that the set of lengths $L_{n}=1-2 a_{n}$ is strictly decreasing with $n$. By construction the string is the set of lengths $L_{n}$, with multiplicity $2^{n}$, given by

$$
L_{1}=1-2 a_{1}, \quad L_{n}=\prod_{i=1}^{n-1} a_{i}\left(1-2 a_{n}\right) .
$$

By construction of the zeta function,

$$
\begin{aligned}
\zeta_{\mathbf{a}}(s) & =\sum_{n=1}^{\infty} 2^{n} L_{n}^{s} \\
& =\sum_{n=1}^{\infty} 2^{n} \prod_{i=1}^{n-1} a_{i}^{s}\left(1-2 a_{n}\right)^{s} \\
& =\sum_{n=1}^{\infty} \exp \left(n \log 2+s\left(\sum_{i=1}^{n-1} \log a_{i}+\log \left(1-2 a_{n}\right)\right)\right),
\end{aligned}
$$

which will be finite provided the real number $s$ is such that

$$
n \log 2+s\left(\sum_{i=1}^{n-1} \log a_{i}+\log \left(1-2 a_{n}\right)\right)<0 .
$$

Thus we have

$$
\zeta_{\mathbf{a}}(s)<\infty, \text { for } s>\limsup _{n \rightarrow \infty} \frac{\log \prod_{i=1}^{n} a_{i}}{n \log 2}=d_{u b} .
$$

The question we address now is the meromorphic continuation. Let us make some more assumptions about our model. Assume that there is a probability distribution $\mathbb{P}$ on $(0,1 / 3)$ and that $\left\{A_{i}\right\}$ is a sequence of independent and identically distributed random variables with law $\mathbb{P}$. In this case we see that

$$
d_{f}=\operatorname{dim}_{H}\left(C_{\mathbf{a}}\right)=d_{l b}=d_{u b}=\operatorname{dim}_{P}\left(C_{\mathbf{a}}\right)=\frac{-\mathbb{E}\left(\log A_{1}\right)}{\log 2}, \mathbb{P} \text {-a.s. }
$$

We consider $\eta(x)$, the number of intervals with length greater than $1 / x$. By the structure of our sequence we have the identity

$$
\eta_{\mathbf{a}}(x)=I_{\left\{L_{1} \geq 1 / x\right\}}+2 \eta_{\sigma(\mathbf{a})}\left(A_{1} x\right) .
$$

As the sequence is i.i.d. we have

$$
\mathbb{E} \eta_{\mathbf{a}}(x)=\mathbb{P}\left(L_{1} \geq 1 / x\right)+2 \mathbb{E} \eta_{\mathbf{a}}\left(A_{1} x\right) .
$$

This can be written as a renewal equation if we set $m(x)=e^{-\gamma x} \mathbb{E} \eta_{\mathbf{a}}\left(e^{x}\right), g(x)=$ $e^{-\gamma x} \mathbb{P}\left(L_{1} \geq e^{-x}\right)$ and choose $\gamma$ such that $\tilde{\mathbb{P}}(d x)=2 e^{-\gamma x} \mathbb{P}\left(-\log A_{1} \in d x\right)$ is a probability measure,

$$
m(x)=g(x)+\int_{0}^{\infty} m(x-y) \tilde{\mathbb{P}}(d y) .
$$

As $g(x)$ is directly Riemann integrable, we can apply the renewal theorem to obtain $m(x) \rightarrow \int_{0}^{\infty} g(x) d x / \int_{0}^{\infty} 2 x e^{-\gamma x} \mathbb{P}\left(\log A_{1} \in d x\right)$ as $x \rightarrow \infty$, giving the growth rate 
of the counting function. Thus we have $\gamma$ defined to be the solution to $2 \mathbb{E} A_{1}^{\gamma}=1$ and as $x \rightarrow \infty$,

$$
\mathbb{E} \eta_{\mathbf{a}}(x) \sim c x^{\gamma}
$$

Our next task is to show that $\eta(x)$ does not grow like its mean and hence we cannot hope to control the zeta function with the mean zeta function as we have done before. We note that the mean zeta function is straightforward to compute as

$$
\zeta_{\mathbf{a}}(s)=L_{1}^{s}+2 A_{1}^{s} \zeta_{\sigma(\mathbf{a})}(s),
$$

and hence

$$
\mathbb{E} \zeta_{\mathbf{a}}(s)=\frac{\mathbb{E}\left(\left(1-2 A_{1}\right)^{s}\right)}{1-\mathbb{E}\left(2 A_{1}^{s}\right)} .
$$

Thus the poles of the zeta function occur at a subset of the complex solutions to $2 \mathbb{E} A_{1}^{s}=1$, whose maximal real solution is not $d_{f}$. We now apply our previous technique and attempt to control the zeta function by using a random variable $W$ and signed measure $V=\eta-W \mathbb{E} \eta$, such that

$$
\zeta_{\mathbf{a}}(s)=W \mathbb{E} \zeta_{\mathbf{a}}(s)+\int_{0}^{\infty} x^{-s} V(d z) .
$$

By construction of the string we have

$$
\eta(x)=2^{n}-1 \text { if }\left\{\prod_{i=1}^{n} A_{i}\left(1-2 A_{n+1}\right)<1 / x \leq \prod_{i=1}^{n-1} A_{i}\left(1-2 A_{n}\right)\right\} .
$$

Taking the subsequence $x_{n}=\left(\prod_{i=1}^{n-1} A_{i}\left(1-2 A_{n}\right)\right)^{-1}$ we have $\eta\left(x_{n}\right)=2^{n}-1$ and by the ergodic theorem

$$
x_{n}^{1 / n}=\exp \left(\frac{1}{n} \sum_{i=1}^{n-1}-\log A_{i}+\frac{1}{n} \log \left(1-2 A_{n}\right)\right) \rightarrow \exp \left(-\mathbb{E}\left(\log A_{1}\right)\right),
$$

as $n \rightarrow \infty$. Now

$$
\eta\left(x_{n}\right)=x_{n}^{n \log 2 / \log x_{n}}-1 \sim x_{n}^{\log 2 /-\mathbb{E}\left(\log A_{1}\right)},
$$

as $n \rightarrow \infty$. We note that this almost sure growth rate $\log 2 /-\mathbb{E}\left(\log A_{1}\right)$ is different from $\gamma$, the mean growth rate. We can make the oscillation precise by using the law of the iterated logarithm (LIL) for i.i.d. random variables to control the size of the fluctuation in the convergence of $\frac{1}{n} \sum_{i=1}^{n} \log A_{i}$.

Note that by the LIL, for all $\epsilon>0$,

$$
\limsup _{n \rightarrow \infty} \frac{1}{n^{1 / 2+\epsilon}}\left|\sum_{i=1}^{n}\left(\log A_{i}-\mathbb{E}\left(\log A_{i}\right)\right)\right|=0 .
$$

Thus, for any $c>0$, there exists $N<\infty$ such that $x_{n} \leq \exp \left(-n \mathbb{E}\left(\log A_{i}\right)+c n^{1 / 2-\epsilon}\right)$ for all $n \geq N, \mathbb{P}$-a.s. Also there will be a subsequence $n_{k}$ such that

$$
x_{n} \geq \exp \left(-n_{k} \mathbb{E}\left(\log A_{i}\right)+c n_{k}^{1 / 2+\epsilon}\right), \quad \forall k .
$$

Thus we cannot control the growth of the counting function $\eta(x)$ by its mean growth rate, or even by its almost sure growth rate, as the fluctuations are too large. Hence our Euler-Maclaurin approach to meromorphic continuation does not work and a different approach would be required to prove results about the complex dimensions of this random fractal string. 


\section{TUBular NEIGHBOURHOODS AND EIGENVALUE ASYMPTOTICS}

We conclude with an application of our results on the complex dimensions of the string to two problems. First we discuss the geometric problem of determining the volume of a tubular neighbourhood of the string. Second we consider the asymptotic growth of the eigenvalue counting function for the Laplace operator on a bounded domain. In this case we regard a fractal string as a one-dimensional domain with a fractal boundary consisting of the Cantor set which is the complement of the string. We will restrict our attention to random recursive fractals satisfying Assumption 3.3 , and to stable strings.

We will extend the results of 14 concerning explicit formulas for the expansion of the zeta function from the deterministic to the random case. We begin with two hypotheses on the growth of the zeta function. First we need two definitions. Let $r: \mathbb{R} \rightarrow[-\infty, \alpha]$ be a bounded Lipschitz continuous function and define the screen $S$ to be the curve $S: t \mapsto r(t)+i t$ for $t \in \mathbb{R}$ and the window $\mathcal{W}=\{s \in \mathbb{C}$ : $R e(s) \geq r(\operatorname{Im}(s))\}$. We will write $D_{\eta}(\mathcal{W})$ for the set of complex dimensions of the string $\eta$ which lie in the window $\mathcal{W}$; these are referred to as the 'visible' complex dimensions.

Assumption 7.1. There exist real constants $\kappa \geq 0$ and $C(\omega)>0$ and a sequence $\left\{T_{n}\right\}_{n \in \mathbb{Z}}$ of real numbers tending to $\pm \infty$ as $n \rightarrow \pm \infty$ with $T_{-n}<0<T_{n}$ for $n \geq 1$ and $\lim _{n \rightarrow \infty} T_{n} /\left|T_{-n}\right|=1$, such that for $\mathbb{P}$-almost every $\omega \in \Omega$,

(H1) For all $n \in \mathbb{Z}$ and all $\sigma \geq r\left(T_{n}\right)$,

$$
\left|\zeta_{\eta(\omega)}\left(\sigma+i T_{n}\right)\right| \leq C(\omega)\left|T_{n}\right|^{\kappa}
$$

(H2) For all $t \in \mathbb{R},|t| \geq 1$,

$$
\left|\zeta_{\eta(\omega)}(r(t)+i t)\right| \leq C(\omega)|t|^{\kappa}
$$

We will show in Lemma 7.5 that there are examples of fractal strings which satisfy this assumption.

We now give a distributional formula for the counting function of the string, a version of [14], Theorem 4.12. We regard the measure $\eta$ generated by the string as a Schwartz distribution. It acts on test functions $\varphi$ by

$$
\langle\eta, \varphi\rangle=\int_{0}^{\infty} \varphi(x) \eta(d x)
$$

We write $\mathcal{P}^{[k]} \eta$ for the $k$-th primitive of the distribution, defined by its action on $\varphi$, through

$$
\left\langle\mathcal{P}^{[k]} \eta, \varphi\right\rangle=\int_{0}^{\infty} \int_{y}^{\infty} \frac{(x-y)^{k-1}}{(k-1) !} \varphi(x) d x \eta(d y)
$$

and $\mathcal{P}^{[0]} \eta=\eta$

Theorem 7.2. Let $\eta$ be a random fractal string satisfying Assumption 7.1 , Then for every $k \in \mathbb{Z}$ the explicit formula for the distribution $\mathcal{P}^{[k]} \eta$ on $(0, \infty)$ is given for 
$\mathbb{P}$-almost all $\omega \in \Omega$ by

$$
\begin{aligned}
\mathcal{P}^{[k]} \eta(x)= & \sum_{z \in D_{\eta(\omega)}(\mathcal{W})} \operatorname{Res}\left(\frac{x^{s+k-1} \zeta_{\eta(\omega)}(s)}{\Gamma(s+k) / \Gamma(s)} ; s=z\right) \\
& +\frac{1}{(k-1) !} \sum_{\substack{j=0 \\
-j \in \mathcal{W} \backslash D_{\eta}(\omega)}}^{k-1}\left(\begin{array}{c}
k-1 \\
j
\end{array}\right)(-1)^{j} x^{-j} \zeta_{\eta}(-j)+R_{\eta(\omega)}^{[k]}(x),
\end{aligned}
$$

where

$$
\left\langle R_{\eta(\omega)}^{[k]}, \varphi\right\rangle=\frac{1}{2 \pi i} \int_{S} \int_{0}^{\infty} \zeta_{\eta(\omega)}(s) \varphi(x) \frac{x^{s+k-1}}{\Gamma(s+k) / \Gamma(s)} d x d s .
$$

Remark 7.3. Extending to the random case, 14, Theorem 4.20, we conclude that under the same assumptions as for Theorem [7.2, the error term $R(x)=R_{\eta(\omega)}^{[k]}(x)$ is given for $\mathbb{P}$-a.e. $\omega$ by $R(x)=O\left(x^{\sigma_{u}+k-1}\right)$ as $x \rightarrow \infty$, where $\sigma_{u}=\sup _{t \in \mathbb{R}} r(t)$. Further, if $r(t)<\sigma_{u}$ for all $t \in \mathbb{R}$ (that is, the screen lies entirely to the left of the line $\left.\operatorname{Re}(s)=\sigma_{u}\right)$, then $R(x)=o\left(x^{\sigma_{u}+k-1}\right)$ as $x \rightarrow \infty$. Note that here, as in 14, Definition 4.22, we write that $R(x)=O\left(x^{\beta}\right)$ (resp. $\left.o\left(x^{\beta}\right)\right)$ as $x \rightarrow \infty$ if $\left\langle R, \phi_{a}\right\rangle=O\left(a^{\beta}\right)\left(\operatorname{resp} . o\left(a^{\beta}\right)\right)$ as $a \rightarrow \infty$, where $\phi_{a}(x)=a^{-1} \phi\left(a^{-1} x\right)$.

We note that the case we will be most concerned with is when $k=1$ and the screen is a vertical line to the right of 0 .

Corollary 7.4. Let $\eta$ be a random fractal string satisfying Assumption 7.1 with screen given by $r(t)=\gamma$. Then $\mathbb{P}$-a.s., for $x \rightarrow \infty$, the counting function satisfies

$$
\eta(x)=\sum_{z \in D_{\eta}(\mathcal{W})} \operatorname{Res}\left(\frac{x^{s} \zeta_{\eta}(s)}{s} ; s=z\right)+o\left(x^{\gamma+\delta}\right),
$$

for every $\delta>0$.

Lemma 7.5. Assumption 7.1 holds for the random recursive fractal string under Assumption 3.3 and it also holds for the stable string.

Proof. This is proved using the control we obtained on the zeta function from the Euler-Maclaurin approach to meromorphic continuation. We recall from (4.4) that the random zeta function can be written as

$$
\zeta_{\eta}(\sigma+i t)=W \mathbb{E} \zeta_{\eta}(\sigma+i t)+\int_{0}^{\infty} x^{-\sigma-i t} V(d x)
$$

Now to control the vertical and horizontal growth of this function we use that

$$
\begin{aligned}
\left|\zeta_{\eta}(\sigma+i t)\right| & \leq W \mathbb{E}\left|\zeta_{\eta}(\sigma+i t)\right|+\int_{0}^{\infty}\left|x^{-\sigma-i t}\right| V(d x) \\
& \leq W \mathbb{E}\left|\zeta_{\eta}(\sigma+i t)\right|+C(\sigma, \omega)
\end{aligned}
$$

for all $\sigma>\alpha-\tau$ and $t \in \mathbb{R}$. The constant $C(\sigma, \omega) \rightarrow 0$ as $\sigma \rightarrow \infty$.

Thus the problem is reduced to that of controlling the growth of the mean zeta function.

(1) The stable string: For the stable string we have an explicit expression for the mean zeta function, and the control we require just follows from the properties of the Gamma function. To prove (H1) we consider $\Gamma(s-\alpha) / \Gamma(s)$. Note that the ratio of two Gamma functions, with the parameter of the numerator of smaller real value than that of the denominator, is bounded as $\operatorname{Re}(s) \rightarrow \infty$. Hence we can choose 
$\kappa=0$ and a suitable random constant $C$ from the sum of the deterministic bound and the random control on the fluctuation term to obtain (H1). For (H2) we note that there are no poles except at $\alpha$ and hence we have the vertical control.

(2) The random recursive string: As we are assuming that the probability space is finite, we can follow the ideas of [14. For a lattice string, the poles of the zeta function occur on a finite number of straight lines, leading to (H2), while the periodicity ensures that we can satisfy (H1). In 14 it is observed that a deterministic non-lattice self-similar string can be approximated by a sequence of lattice strings. As the structure of our mean zeta function is the same as the deterministic case, we can also approximate the non-lattice case by the lattice case. Thus a suitable screen can be constructed which gives a bound on the growth of the mean zeta function (see [14, Theorem 2.33) and hence the result we require.

We now conclude by stating some results that follow from our earlier theorems.

Let $V(\epsilon)$ denote the volume of the inner $\epsilon$-neighbourhood of the string $\mathcal{L}$. That is, we recall that the string is defined as a bounded open subset $U$ of the real line and set $V(\epsilon)=\left|U_{\epsilon}\right|$, the one-dimensional Lebesgue measure of the (one-sided) $\epsilon$ neighbourhood of the boundary of the string, $U_{\epsilon}=\{x \in U$ : $\operatorname{dist}(x, \partial U)<\epsilon\}$. This can be expressed as

$$
V(\epsilon)=\sum_{j: l_{j} \geq 2 \epsilon} 2 \epsilon+\sum_{j: l_{j}<2 \epsilon} l_{j}=\left\langle v_{\epsilon}, \eta\right\rangle,
$$

where $v_{\epsilon}(x)=2 \epsilon$ for $x \leq 1 /(2 \epsilon)$ and $v_{\epsilon}(x)=1 / x$ for $x>1 /(2 \epsilon)$.

The following 'random tube formula' is obtained by a simple adjustment of [14], Theorem 6.1, to the random string setting.

Theorem 7.6. Under the hypotheses of Theorem 7.2, the volume of the tubular neighbourhood of the boundary of the random string $\eta$ is given as $\epsilon \rightarrow 0^{+}$, for $\mathbb{P}$-almost all $\omega \in \Omega$, by

$$
\begin{aligned}
& V(\epsilon)=\sum_{z \in D_{\eta(\omega)}(\mathcal{W})} \operatorname{res}\left(\frac{\zeta_{\eta(\omega)}(s)(2 \epsilon)^{1-s}}{s(1-s)} ; s=z\right) \\
& +2 \epsilon \zeta_{\eta(\omega)}(0) I_{\left\{0 \in \mathcal{W} \backslash D_{\eta(\omega)}(\mathcal{W})\right\}}+R_{\eta(\omega)}(\epsilon),
\end{aligned}
$$

where

$$
R_{\eta(\omega)}(\epsilon)=\frac{1}{2 \pi i} \int_{S}(2 \epsilon)^{1-s} \zeta_{\eta(\omega)}(s) \frac{d s}{s(1-s)} .
$$

The remainder satisfies $R_{\eta}(\epsilon)=O\left(\epsilon^{1-\sigma_{u}}\right)$ with $\sigma_{u}$ as given in Remark 7.3 .

Remark 7.7. If $z$ is a simple pole of $\zeta_{\eta}(s)$, then

$$
\operatorname{res}\left(\frac{\zeta_{\eta(\omega)}(s)(2 \epsilon)^{1-s}}{s(1-s)} ; s=z\right)=\operatorname{res}\left(\zeta_{\eta(\omega)}(s) ; s=z\right) \frac{(2 \epsilon)^{1-z}}{z(1-z)} .
$$

We next apply Theorem 7.6 and adapt the techniques of [14, Section 6.3.1, to the random strings we considered in Sections 4 and 5 . We recall that $W$ is the limit random variable arising in the general branching process and $L$ is the limit random variable arising from the random sequence with Poisson-Dirichlet distribution. 
Theorem 7.8. (1) The volume of the tubular neighbourhood of the random recursive string $\mathcal{L}$ is given $\mathbb{P}$-almost surely by (17.1), where $\mathcal{W}=\{s: \operatorname{Re}(s)>\alpha-\tau\}$, with $\alpha=d_{f}$ and $\tau$ as in Theorem 4.5, and $R(\epsilon)=o\left(\epsilon^{1-\alpha+\tau-\delta}\right)$, for every fixed $\delta>0$ as $\epsilon \rightarrow 0^{+}$. Moreover, for a non-lattice random recursive string, we have

$V(\epsilon)=W \operatorname{Res}(\mathbb{E}(\zeta(s)) ; s=\alpha)(2 \epsilon)^{1-\alpha} \alpha(1-\alpha)+o\left(\epsilon^{1-\alpha}\right)$, as $\epsilon \rightarrow 0^{+}, \mathbb{P}$-a.s.

(2) For the $\alpha$-stable string, the volume $V(\epsilon)$ of a tubular neighbourhood, for every fixed $\delta>0$, is given by

$$
V(\epsilon)=\frac{L(2 \epsilon)^{1-\alpha}}{(1-\alpha)}+o\left(\epsilon^{1-\alpha / 2-\delta}\right), \text { as } \epsilon \rightarrow 0^{+}, \quad \mathbb{P}_{\alpha}-a . s .
$$

A consequence of this result is that, in the case of the stable string and the (strongly) non-lattice random recursive string, the boundary of the string, that is, the associated random Cantor set, is almost surely Minkowski measurable. Some results in this direction appear in 7 .

We now turn to the eigenvalue counting function. In the case of a random fractal, the result of [9] shows that the coefficient of the leading term in the asymptotic expansion of the counting function can be a random constant. As we are considering a fractal boundary value problem, we observe a similar result for the second order term. As the string consists of a sequence of one-dimensional intervals, it is easy to write down the eigenvalues (up to a normalization) for the Dirichlet Laplacian as $k l_{j}^{-1}$ for $k, j=1,2, \ldots$. Thus there is a simple relationship between the spectral zeta function, $\zeta_{s p}(s)$, for the string and the geometric zeta function as

$$
\zeta_{s p}(z)=\sum_{j=1}^{\infty} \lambda_{j}^{s}=\sum_{j, k=1}^{\infty} k^{s} l_{j}^{s}=\zeta_{r}(z) \zeta_{\eta}(z),
$$

where $\zeta_{r}(z)$ is the usual Riemann zeta function. Hence we can transfer any results about the geometry of the string through to its spectrum.

Let $N_{s p}(x)$ denote the number of eigenvalues of the Laplacian of the fractal string with value less than $x$.

Theorem 7.9. The eigenvalue counting function for the non-lattice random recursive fractal string is given by

$$
N_{s p}(x)=x+W \zeta_{r}(\alpha) \operatorname{Res}\left(\mathbb{E} \zeta_{\eta}(s) ; s=\alpha\right) \frac{x^{\alpha}}{\alpha}+o\left(x^{\alpha}\right), \text { as } x \rightarrow \infty, \mathbb{P} \text {-a.s. }
$$

For the $\alpha$-stable string

$$
N_{s p}(x)=x+L \zeta_{r}(\alpha) x^{\alpha}+o\left(x^{\alpha / 2+\epsilon}\right), \text { as } x \rightarrow \infty, \mathbb{P}_{\alpha} \text {-a.s. }
$$

In closing we mention an open problem that naturally arises from the results presented here. In 14 it was proposed that the definition of fractality should be the existence of at least one non-real complex dimension (with positive real part). As we have seen in Section 5 there are natural random strings for which this is not the case. However we have not been able to establish meromorphic continuation of the geometric zeta functions of these strings to all of $\mathbb{C}$. This raises the question of whether there is a natural boundary for such strings beyond which the zeta function cannot be extended. 


\section{ACKNOWLEDGEMENT}

The authors would like to thank the organisers of the Isaac Newton Institute programme 'The mathematics and applications of fractals', where this work was initiated.

\section{AdDed AFter Posting}

It may be helpful to the reader to specify that in either the introduction or in Theorem 7.9 (and the text preceding it), we have used the following implicit convention. As in reference [14, $N=N(x)$ really denotes the normalized frequency (rather than eigenvalue) counting function for the Dirichlet Laplacian on the bounded open set $U$; that is, we have the relation $x=\sqrt{\lambda} / \pi$, where $\lambda$ is the eigenvalue parameter.

\section{REFERENCES}

[1] M.T. Barlow and B.M. Hambly, Transition density estimates for Brownian motion on scale irregular Sierpinski gaskets. Ann. Inst. H. Poincaré Probab. Statist. 33, 531-557, 1997. MR:1473565 (98k:60137)

[2] M.V. Berry, Distribution of modes in fractal resonators, in: Structural Stability in Physics (eds.: W. Güttinger and H. Eikemeier), Springer-Verlag, Berlin, 1979, pp. 51-53. MR0556688

[3] N.H. Bingham and R.A. Doney, Asymptotic properties of supercritical branching processes. II. Crump-Mode and Jirina processes. Adv. Appl. Probab. 7, 66-82, 1975. MR0378125|(51:14294)

[4] J. Brossard and R. Carmona, Can one hear the dimension of a fractal?, Commun. Math. Phys. 104, 103-122, 1986. MR0834484 (87h:58218)

[5] W.D. Evans and D.J. Harris, Fractals, trees and the Neumann Laplacian. Math. Ann. 296, 493-527, 1993. MR1225988(94k:35218)

[6] K.J. Falconer, Random fractals. Math. Proc. Cambridge Philos. Soc. 100, 559-582, 1986. MR0857731 (88e:28005)

[7] D. Gatzouras, Lacunarity of self-similar and stochastically self-similar sets. Trans. Amer. Math. Soc. 352, 1953-1983, 2000. MR.1694290 (2000j:28006)

[8] B.M. Hambly, Brownian motion on a random recursive Sierpinski gasket. Ann. Probab. 25, 1059-1102, 1997. MR.1457612 (98i:60072)

[9] B.M. Hambly, On the asymptotics of the eigenvalue counting function for random recursive Sierpinski gaskets. Probab. Theory Related Fields 117, 221-247, 2000. MR1771662 (2002h:28011)

[10] J. Kigami, Analysis on Fractals. Cambridge University Press, 2001. MR1840042 (2002c:28015)

[11] M.L. Lapidus, Fractal drum, inverse spectral problems for elliptic operators and a partial resolution of the Weyl-Berry conjecture. Trans. Amer. Math. Soc. 325, 465-529, 1991. MR0994168 (91j:58163)

[12] M.L. Lapidus and C. Pomerance, The Riemann zeta-function and the one-dimensional WeylBerry conjecture for fractal drums. Proc. London Math. Soc. 66, 41-69, 1993. MR.1189091 (93k:58217)

[13] M.L. Lapidus and C. Pomerance, Counterexamples to the modified Weyl-Berry conjecture on fractal drums. Math. Proc. Cambridge Philos. Soc. 119, 167-178, 1996. MR1356166 (96h:58175)

[14] M.L. Lapidus and M. van Frankenhuysen, Fractal Geometry and Number Theory. Birkhäuser, Boston, 2000. MR:1726744(2001b:11079)

[15] M.L. Lapidus and M. van Frankenhuysen, Fractality, self-similarity and complex dimensions. in Fractal Geometry and Applications: a Jubilee of Benoit Mandelbrot, Proc. Sympos. Pure Math., vol. 72, Part 1, Amer. Math. Soc., Providence, R.I., 2004, pp. 349-372.

[16] T. Lindvall, Lectures on the Coupling Method, Wiley, Chichester, 1992. MR:1180522 (94c:60002)

[17] R.D. Mauldin and S.C. Williams, Random recursive constructions: asymptotic geometric and topological properties. Trans. Amer. Math. Soc. 295, 325-346, 1986. MR0831202 (87j:60027) 
[18] O. Nerman, On the convergence of supercritical general (C-M-J) branching processes. Zeit. Wahr. 57, 365-395, 1981. MR0629532 (82m:60104)

[19] J. Pitman and M. Yor, The two parameter Poisson-Dirichlet distribution derived from a stable subordinator. Ann. Probab. 25, 855-900, 1997. MR1434129 (98f:60147)

[20] C.J. Stone, On moment generating functions and renewal theory. Ann. Math. Stat. 36, 12981301, 1965. MR0179857 (31:4097)

Mathematical Institute, University of Oxford, 24-29 St Giles, Oxford OX1 3LB, UNITED KINGDOM

E-mail address: hambly@maths.ox.ac.uk

Department of Mathematics, University of California, Riverside, California 925210135

E-mail address: lapidus@math.ucr.edu 